\title{
Antichains on Three Levels *
}

\author{
Paulette Lieby \\ Autonomous Systems and Sensing Technologies Programme \\ National ICT Australia, Locked Bag 8001 \\ Canberra, ACT 2601, Australia \\ Paulette.Lieby@nicta.com.au
}

Submitted: Mar 8, 2003; Accepted: Jun 18, 2004; Published: Jul 29, 2004

MR Subject Classification: 05D99

\begin{abstract}
An antichain is a collection of sets in which no two sets are comparable under set inclusion. An antichain $\mathcal{A}$ is flat if there exists an integer $k \geq 0$ such that every set in $\mathcal{A}$ has cardinality either $k$ or $k+1$. The size of $\mathcal{A}$ is $|\mathcal{A}|$ and the volume of $\mathcal{A}$ is $\sum_{A \in \mathcal{A}}|A|$. The flat antichain theorem states that for any antichain $\mathcal{A}$ on $[n]=\{1,2, \ldots, n\}$ there exists a flat antichain on $[n]$ with the same size and volume as $\mathcal{A}$. In this paper we present a key part of the proof of the flat antichain theorem, namely we show that the theorem holds for antichains on three consecutive levels; that is, in which every set has cardinality $k+1, k$ or $k-1$ for some integer $k \geq 1$. In fact we prove a stronger result which should be of independent interest. Using the fact that the flat antichain theorem holds for antichains on three consecutive levels, together with an unpublished result by the author and A. Woods showing that the theorem also holds for antichains on four consecutive levels, Á. Kisvölcsey completed the proof of the flat antichain theorem. This proof is to appear in Combinatorica.

The squashed (or colex) order on sets is the set ordering with the property that the number of subsets of a collection of sets of size $k$ is minimised when the collection consists of an initial segment of sets of size $k$ in squashed order. Let $p$ be a positive integer, and let $\mathcal{A}$ consist of $p$ subsets of $[n]$ of size $k+1$ such that, in the squashed order, these subsets are consecutive. Let $\mathcal{B}$ consist of any $p$ subsets of $[n]$ of size $k-1$. Let $\left|\triangle_{N} \mathcal{A}\right|$ be the number of subsets of size $k$ of the sets in $\mathcal{A}$ which are not subsets of any set of size $k+1$ preceding the sets in $\mathcal{A}$ in the squashed order. Let $|\nabla \mathcal{B}|$ be the number of supersets of size $k$ of the sets in $\mathcal{B}$. We show that $\left|\triangle_{N} \mathcal{A}\right|+|\nabla \mathcal{B}|>2 p$. We call this result the 3-levels result. The 3-levels result implies that the flat antichain theorem is true for antichains on at most three, consecutive, levels.
\end{abstract}

*This research was done while at Charles Darwin University, NT 0909, Australia. 


\section{Introduction}

\subsection{Definitions and Notation}

\section{Sets, Collections of Sets, and Orderings on Sets}

Throughout the paper the universal set is the finite set $\{1, \ldots, n\}$ which is denoted by $[n]$. The size or cardinality of a set $B$ is $|B|$. If $|B|=k$, then $B$ is a $k$-set or a $k$-subset. Alternatively we say that $B$ is a set on level $k$. The collection of all the $k$-subsets of $[n]$ is denoted by $[n]^{k}$.

When no ambiguity arises the braces are left out when writing sets: The set $\{a, b, c\}$ may be written $a b c$.

For sets $A$ and $B$, the set difference of $A$ and $B$ is $A \backslash B=\{i: i \in A, i \notin B\}$. The symmetric difference of $A$ and $B$ is $A+B=(A \backslash B) \cup(B \backslash A)$. The complement of a subset $B$ of $[n]$ is $B^{\prime}=[n] \backslash B$.

Let $\mathcal{B}$ be a collection of subsets of $[n]$. The size or cardinality of $\mathcal{B}$ is $|\mathcal{B}|$ and its volume is $V(\mathcal{B})=\sum_{B \in \mathcal{B}}|B|$. The average set size of $\mathcal{B}$ is $\overline{\mathcal{B}}=V(\mathcal{B}) /|\mathcal{B}|$. The complement of $\mathcal{B}$ is $\mathcal{B}^{\prime}=\left\{B: B^{\prime} \in \mathcal{B}\right\}$. If the sets in $\mathcal{B}$ are ordered, then the sets in $\mathcal{B}^{\prime}$ inherit the same ordering from $\mathcal{B}$. The collection of the $i$-sets in $\mathcal{B}$ is denoted by $\mathcal{B}^{(i)}=\{B$ : $B \in \mathcal{B},|B|=i\}$. The parameters of $\mathcal{B}$ are the integers $p_{i}=\left|\mathcal{B}^{(i)}\right|, 0 \leq i \leq n$, and its levels are the integers $i$ for which $p_{i}>0$.

The collection $\mathcal{B}$ is flat if for all $B \in \mathcal{B},|B|=\lfloor\overline{\mathcal{B}}\rfloor$ or $|B|=\lfloor\overline{\mathcal{B}}\rfloor+1$. That is, $\mathcal{B}$ is flat if it has at most two levels, and those levels are consecutive.

A partition of $\mathcal{B}$ is a collection of pairwise disjoint sub-collections of $\mathcal{B}$ whose union is $\mathcal{B}$. That is, the collection $\pi_{1}=\left\{\mathcal{B}_{1}, \mathcal{B}_{2}, \ldots, \mathcal{B}_{m}\right\}$ with $\mathcal{B}_{i} \cap \mathcal{B}_{j}=\emptyset, 1 \leq i<j \leq m$, and $\bigcup_{i=1}^{m} \mathcal{B}_{i}=\mathcal{B}$ is a partition of $\mathcal{B}$. Note that in this definition of a partition of $\mathcal{B}$, the sub-collections are allowed to be empty.

Let $L$ be a set such that $B \cap L=\emptyset$ for all $B \in \mathcal{B}$, and $b<l$ for all $b \in B \in \mathcal{B}$ and all $l \in L$. Then $\mathcal{B} \uplus L$ is defined to be $\mathcal{B} \uplus L=\{B \cup L, B \in \mathcal{B}\}$.

Example 1.1. Let $\mathcal{B}=\{1,13,23\}$ and $L=\{56\}$. Then $\mathcal{B} \uplus L=\{156,1356,2356\}$.

A total order on sets, the squashed order, denoted by $\leq_{S}$, is defined by: If $A$ and $B$ are any sets, then $A \leq_{S} B$ if the largest element in $A+B$ is in $B$ or if $A=B$. We write $A<_{S} B$ or $B>_{S} A$ if $A \leq_{S} B$ and $A \neq B$. If $\mathcal{B}$ is a collection of sets in squashed order, we write $A<_{S} \mathcal{B}$ or $\mathcal{B}>_{S} A$ if $A<_{S} B$ for all $B \in \mathcal{B}$, and $A>_{S} \mathcal{B}$ or $\mathcal{B}<_{S} A$ if $A>_{S} B$ for all $B \in \mathcal{B}$.

The reverse of the squashed order for subsets of $[n]$ is called the antilexicographic order and is denoted by $\leq_{A}$. That is, $A \leq_{A} B$ implies that the largest element of $A+B$ is in $A$ 
or $A=B$.

Example 1.2. The first ten 3-sets in squashed order are: 123, 124, 134, 234, 125, 135, $235,145,245,345$.

The first 5 3-subsets of [5] in antilexicographic order are: 345, 245, 145, 235, 135.

$F_{n, k}(p)$ and $L_{n, k}(p)$ denote the collections of the first $p$ and the last $p k$-subsets of $[n]$ in squashed order respectively. $C_{n, k}(p)$ denotes any collection of $p$ consecutive $k$-subsets of $[n]$ in squashed order. If a collection $C_{n, k}(p)$ comes immediately after the collection $F_{n, k}(m)$ in squashed order, then it is denoted by $N_{n, k}^{m}(p)$. If a collection $C_{n, k}(p)$ comes immediately before the collection $L_{n, k}(m)$ in squashed order, then it is denoted by $P_{n, k}^{m}(p)$. Note that the use of the notation $F_{n, k}(p), L_{n, k}(p), \ldots$, implicitly assumes that $0 \leq k \leq n$ and $p \leq\left(\begin{array}{l}n \\ k\end{array}\right)$.

Let $\mathcal{B}$ be a collection of $p$ sets in squashed order. If $\mathcal{B}=F_{n, k}(p)$ we say that $\mathcal{B}$ is an initial segment of $k$-sets in squashed order or that $\mathcal{B}$ is a terminal segment of $k$-sets in antilexicographic order. If $\mathcal{B}=L_{n, k}(p)$ we say that $\mathcal{B}$ is a terminal segment of $k$-subsets of $[n]$ in squashed order or that $\mathcal{B}$ is an initial segment of $k$-subsets of $[n]$ in antilexicographic order. Finally, $F(p, \mathcal{B})$ and $L(p, \mathcal{B})$ respectively denote the first and the last $p$ sets of $\mathcal{B}$.

\section{Shadows and Shades}

Let $B$ be a $k$-subset of $[n]$. The shadow of $B$ is $\triangle B=\{D: D \subset B,|D|=k-1\}$ and its shade is $\nabla B=\{D \subseteq[n]: D \supset B,|D|=k+1\}$. The new-shadow of $B$ is $\triangle_{N} B=\left\{D: D \in \triangle B, D \notin \triangle C\right.$ for all $\left.C<_{S} B\right\}$. That is, $\triangle_{N} B$ is the collection of the $(k-1)$-sets which belong to the shadow of $B$ but not to the shadow of any $k$-set which precedes $B$ in squashed order. In other words, if $B$ is the $p$-th set in squashed order, the new-shadow of $B$ can be thought of as being the contribution of $B$ to the shadow of the first $p k$-sets in squashed order. Similarly, the new-shade of $B$ is $\nabla_{N} B=\{D: D \in$ $\nabla B, D \notin \nabla C$ for all $\left.C>_{S} B\right\}$. That is, $\nabla_{N} B$ consists of the $(k+1)$-sets which are in the shade of $B$ but not in the shade of any $k$-set which follows $B$ in squashed order.

Let $\mathcal{B}$ be a collection of $k$-subsets of $[n]$. The shadow of $\mathcal{B}$ is $\triangle \mathcal{B}=\bigcup_{B \in \mathcal{B}} \triangle B$ and its shade is $\nabla \mathcal{B}=\bigcup_{B \in \mathcal{B}} \nabla B$. The new-shadow of $\mathcal{B}$ is $\triangle_{N} \mathcal{B}=\bigcup_{B \in \mathcal{B}} \triangle_{N} B$ and its new-shade is $\nabla_{N} \mathcal{B}=\bigcup_{B \in \mathcal{B}} \nabla_{N} B$.

Example 1.3. Let $[n]=[5]$. For each 3 -subset of [5], we list the sets in its new-shadow and the sets in its new-shade. The 3 -sets are listed in squashed order.

\begin{tabular}{r|llllllllll}
$B$ & 123 & 124 & 134 & 234 & 125 & 135 & 235 & 145 & 245 & 345 \\
$\triangle_{N} B$ & $12,13,23$ & 14,24 & 34 & - & 15,25 & 35 & - & 45 & - & - \\
$\nabla_{N} B$ & - & - & - & 1234 & - & - & 1235 & - & 1245 & 1345,2345
\end{tabular}




\section{Antichains}

An antichain on $[n]$ is a set of incomparable elements in the Boolean lattice of order $n$, the subsets of $[n]$ ordered by inclusion. Let $\mathcal{A}$ be an antichain on $[n]$ with largest and smallest set size $h$ and $l$ respectively. For $l \leq i \leq h$, let $p_{i}=\left|\mathcal{A}^{(i)}\right|$ be the number of subsets of size $i$ in $\mathcal{A}$. The antichain $\mathcal{A}$ is squashed if, for $i=h, h-1, \ldots, l$,

$$
\mathcal{A}^{(i)}=N_{n, i}^{q_{i}}\left(p_{i}\right)
$$

where $q_{h}=0$, and for $i<h, q_{i}=\left|\triangle F_{n, i+1}\left(q_{i+1}+p_{i+1}\right)\right|$. That is, the sets in $\triangle F_{n, i+1}\left(q_{i+1}+\right.$ $\left.p_{i+1}\right) \cup \mathcal{A}^{(i)}$ form an initial segment of $q_{i}+p_{i} i$-sets in squashed order.

\subsection{The Main Results}

This paper presents two main results. The first concerns the number of subsets and supersets of certain collections of subsets of a finite set $[n]$.

Theorem 1.4 (The 3-levels result). Let $n, k$, and $p$ be positive integers with $1 \leq k<n$ and $p \leq \min \left\{\left(\begin{array}{c}n \\ k+1\end{array}\right),\left(\begin{array}{c}n \\ k-1\end{array}\right)\right\}$. Let $\mathcal{A}$ consist of $p$ subsets of $[n]$ of size $k+1$ such that, in the squashed order, these subsets are consecutive. Let $\mathcal{B}$ consist of any $p$ subsets of $[n]$ of size $k-1$. Then

$$
\left|\triangle_{N} \mathcal{A}\right|+|\nabla \mathcal{B}|>2 p \text {. }
$$

An alternative form of the 3-levels result theorem is given by the theorem below. That both theorems are equivalent can be seen by application of Corollary 2.7 and Theorem 2.9 (see Section 2).

Theorem 1.5. Let $n, k$, and $p$ be positive integers with $1 \leq k<n$ and $p \leq \min \left\{\left(\begin{array}{c}n \\ k+1\end{array}\right),\left(\begin{array}{c}n \\ k-1\end{array}\right)\right\}$. Then

$$
\left|\triangle_{N} L_{n, k+1}(p)\right|+\left|\nabla_{N} L_{n, k-1}(p)\right|>2 p \text {. }
$$

Exact values for $\left|\triangle_{N} L_{n, k+1}(p)\right|$ and $\left|\nabla_{N} L_{n, k-1}(p)\right|$ are known (see [1, 11]) but these values are not always practical to use in an analytical sense. It is in this sense that we regard Theorem 1.5 as an important result as it provides a simple lower bound for the sum $\left|\triangle_{N} L_{n, k+1}(p)\right|+\left|\nabla_{N} L_{n, k-1}(p)\right|$.

Theorem 1.4 is a key part of the proof of the flat antichain theorem.

Theorem 1.6 (The flat antichain theorem). For any antichain $\mathcal{A}$ on $[n]$ there exists a flat antichain $\mathcal{A}^{*}$ on $[n]$ such that $\left|\mathcal{A}^{*}\right|=|\mathcal{A}|$ and $V\left(\mathcal{A}^{*}\right)=V(\mathcal{A})$.

The flat antichain theorem has been conjectured by the author in 1994 [10]. The theorem is known to hold for $\mathcal{A}$ when $\overline{\mathcal{A}}$ is an integer (see [13]) or when $\overline{\mathcal{A}} \leq 3$ (see [14]). Theorem 1.4 is used to show that the flat antichain theorem holds when the antichain has sets on at most three consecutive levels. This is the second major result in this paper. 
Theorem 1.7. Let $\mathcal{A}$ be an antichain on $[n]$ with parameters $p_{i}$ and let $h$ and $l$ respectively be the largest and smallest integer for which $p_{i} \neq 0$. Assume that $h=k+1$ and $l=k-1$ for some $k \in \boldsymbol{Z}^{+}$. Then the flat antichain theorem holds for $\mathcal{A}$.

Proof. Without loss of generality, $\mathcal{A}$ can be assumed to be squashed (see Theorem 2.8 below). Assume that $p_{k+1} \geq p_{k-1}$ and let $\mathcal{C}$ consist of the last $p_{k-1}$ sets of $\mathcal{A}^{(k+1)}$, that is, $\mathcal{C}=L\left(p_{k-1}, \mathcal{A}^{(k+1)}\right)$. By Theorem $1.4\left|\triangle_{N} \mathcal{C}\right|+\left|\nabla \mathcal{A}^{(k-1)}\right|>2 p_{k-1}$. Thus there exists a flat antichain on $[n]$ consisting of $p_{k+1}-p_{k-1}(k+1)$-sets and $p_{k}+2 p_{k-1} k$-sets. The case $p_{k+1}<p_{k-1}$ is dealt with in a similar manner.

Using Theorem 1.7 and an additional result by the author and A. Woods [11] showing that Theorem 1.6 holds for antichains on four consecutive levels, A. Kisvölcsey [8] completed the proof of the flat antichain theorem and thus showed the validity of the original conjecture.

To prove the 3-levels result we prove its equivalent form as given by Theorem 1.5; this proof is long and complex. Section 2 provides the background material needed in the paper. The proof of Theorem 1.5 is split into three parts A, B and C, to be found in Sections 3, 4 and 5 respectively. Parts A and B consider the cases when $k \leq \frac{n}{2}$, and Part C proves Theorem 1.5 in the case $k>\frac{n}{2}$. See Figure 1 page 9 for an outline of the proof. The paper ends with Section 6 which discusses some possible alternative proofs of Theorem 1.5.

The author is deeply grateful to two (anonymous) referees for their thorough and comprehensive review; their keen interest was very encouraging. Warm thanks to G. Brown and B. McKay for reading the successive drafts and providing useful feedback. A fully detailed proof is available at http://cs.anu.edu.au/ bdm/lieby.html.

\section{Background Material}

Most of the material surveyed here can be found in [1]. In the course of the paper, no explicit reference will be made to the results cited -which are standard in Sperner theory, except in a few specific instances.

\subsection{Simple Facts}

Let $A$ and $B$ be two sets such that $A \leq_{S} B$. Since $A+B=A^{\prime}+B^{\prime}, A \leq_{S} B$ if and only if $B^{\prime} \leq_{S} A^{\prime}$ and $A^{\prime} \leq_{A} B^{\prime}$. Thus, $\mathcal{B}$ is a collection of sets in squashed order if and only if $\mathcal{B}^{\prime}$ is a collection of sets in antilexicographic order. In particular,

$$
\left(F_{n, k}(p)\right)^{\prime}=L_{n, n-k}(p) .
$$


The self-duality of the Boolean lattice enables us to write that $(\triangle B)^{\prime}=\nabla B^{\prime},(\triangle \mathcal{B})^{\prime}=$ $\nabla \mathcal{B}^{\prime},\left(\triangle_{N} B\right)^{\prime}=\nabla_{N} B^{\prime}$, and $\left(\triangle_{N} \mathcal{B}\right)^{\prime}=\nabla_{N} \mathcal{B}^{\prime}$. In particular,

\section{Lemma 2.1.}

$$
\left|\triangle F_{n, k}(p)\right|=\left|\nabla L_{n, n-k}(p)\right|, \text { and }\left|\triangle_{N} L_{n, k}(p)\right|=\left|\nabla_{N} F_{n, n-k}(p)\right| .
$$

The squashed order is independent of the universal set. This implies that $F_{n, k}(p)=$ $F_{n^{\prime}, k}(p)$ for any $n^{\prime}$ such that $p \leq\left(\begin{array}{c}n^{\prime} \\ k\end{array}\right)$.

Given the definition of $F_{n, k}, C_{n, k}$ and $L_{n, k}$, it is easy to see that $F_{n, k}\left(\left(\begin{array}{l}n \\ k\end{array}\right)\right)=C_{n, k}\left(\left(\begin{array}{l}n \\ k\end{array}\right)\right)=$ $L_{n, k}\left(\left(\begin{array}{l}n \\ k\end{array}\right)\right)=[n]^{k}$.

It follows that

$$
\left|\triangle F_{n, k}\left(\left(\begin{array}{l}
n \\
k
\end{array}\right)\right)\right|=\left(\begin{array}{c}
n \\
k-1
\end{array}\right) \text {, and }\left|\nabla L_{n, k}\left(\left(\begin{array}{l}
n \\
k
\end{array}\right)\right)\right|=\left(\begin{array}{c}
n \\
k+1
\end{array}\right) .
$$

The next observations follow from the definitions of the new-shadow and the new-shade. Trivially, $\triangle_{N} F_{n, k}(p)=\triangle F_{n, k}(p)$ and $\nabla_{N} L_{n, k}(p)=\nabla L_{n, k}(p)$. If $\mathcal{A}$ and $\mathcal{B}$ are two collections of $k$-sets such that $\mathcal{A} \cap \mathcal{B}=\emptyset$, then $\left|\triangle_{N}(\mathcal{A} \cup \mathcal{B})\right|=\left|\triangle_{N} \mathcal{A}\right|+\left|\triangle_{N} \mathcal{B}\right|$ and $\left|\nabla_{N}(\mathcal{A} \cup \mathcal{B})\right|=\left|\nabla_{N} \mathcal{A}\right|+\left|\nabla_{N} \mathcal{B}\right|$. Also,

$$
\begin{aligned}
& \left|\triangle_{n, k}\left(p_{1}+p_{2}\right)\right|=\left|\triangle F_{n, k}\left(p_{1}\right)\right|+\left|\triangle_{N} N_{n, k}^{p_{1}}\left(p_{2}\right)\right|, \\
& \left|\triangle_{N} L_{n, k}\left(p_{1}+p_{2}\right)\right|=\left|\triangle_{N} L_{n, k}\left(p_{1}\right)\right|+\left|\triangle_{N} P_{n, k}^{p_{1}}\left(p_{2}\right)\right|, \\
& \left|\nabla_{N} F_{n, k}\left(p_{1}+p_{2}\right)\right|=\left|\nabla_{N} F_{n, k}\left(p_{1}\right)\right|+\left|\nabla_{N} N_{n, k}^{p_{1}}\left(p_{2}\right)\right|, \\
& \left|\nabla L_{n, k}\left(p_{1}+p_{2}\right)\right|=\left|\nabla L_{n, k}\left(p_{1}\right)\right|+\left|\nabla_{N} P_{n, k}^{p_{1}}\left(p_{2}\right)\right| .
\end{aligned}
$$

\subsection{Some Isomorphism Results}

The three lemmas below are obtained by establishing an isomorphism between a collection of $p$ subsets of $[n]$ in squashed order and a collection of $p$ subsets of $[n-i]$ in squashed order for $0<i<n$. This is possible when $p$ is small.

Lemma 2.2. Let $0 \leq i \leq n-k$ and $p \leq\left(\begin{array}{c}n-i \\ k\end{array}\right)$. Then the collections $F_{n, k}(p)$ and $F_{n-i, k}(p)$ are isomorphic and

$$
\begin{aligned}
& \left|\triangle_{N} F_{n, k}(p)\right|=\left|\triangle_{N} F_{n-i, k}(p)\right|, \text { and } \\
& \left|\nabla_{N} F_{n, k}(p)\right|=\left|\nabla_{N} F_{n-i, k}(p)\right| .
\end{aligned}
$$

Lemma 2.3. Let $0 \leq k \leq n$ and let $\mathcal{B}$ be a collection of consecutive $k$-subsets of $[n]$ in squashed order. Assume that $\mathcal{B}=\mathcal{C} \uplus L$ for some $L \subseteq[n]$. Then $\mathcal{B}$ and $\mathcal{C}$ are isomorphic and

$$
\begin{aligned}
& \left|\triangle_{N} \mathcal{B}\right|=\left|\triangle_{N} \mathcal{C}\right|, \text { and } \\
& \left|\nabla_{N} \mathcal{B}\right|=\left|\nabla_{N} \mathcal{C}\right| .
\end{aligned}
$$


Lemma 2.4. Let $0 \leq i \leq k$ and $p \leq\left(\begin{array}{c}n-i \\ k-i\end{array}\right)$. Then the collections $L_{n, k}(p)$ and $L_{n-i, k-i}(p)$ are isomorphic and

$$
\begin{aligned}
\left|\triangle_{N} L_{n, k}(p)\right| & =\left|\triangle_{N} L_{n-i, k-i}(p)\right|, \text { and } \\
\left|\nabla_{N} L_{n, k}(p)\right| & =\left|\nabla_{N} L_{n-i, k-i}(p)\right| .
\end{aligned}
$$

\subsection{Bounds for Shadows and Shades}

Sperner's lemma below gives a lower bound for the sizes of the shadow and the shade of a collection $\mathcal{B}$. The proof of when equality holds in the lemma can be found in $[2, \mathrm{p} .12]$.

Lemma 2.5 (Sperner's lemma, Sperner [15]). Let $\mathcal{B}$ be a collection of $k$-subsets of $[n]$. Then

$$
|\triangle \mathcal{B}| \geq \frac{k}{n-k+1}|\mathcal{B}| \quad \text { if } k>0
$$

and

$$
|\nabla \mathcal{B}| \geq \frac{n-k}{k+1}|\mathcal{B}| \quad \text { if } k<n \text {. }
$$

Equality holds if and only if $\mathcal{B}$ consists of all the $\left(\begin{array}{l}n \\ k\end{array}\right) k$-sets.

The next theorem by Kruskal and Katona shows a very important property of the squashed order.

Theorem 2.6 (Kruskal [9], Katona [7]). Let $\mathcal{B}$ be a collection of $p$-subsets of $[n]$. Then

$$
|\triangle \mathcal{B}| \geq\left|\triangle F_{n, k}(|\mathcal{B}|)\right|
$$

Equality holds when $\mathcal{B}$ is an initial segment of $k$-sets in squashed order.

This theorem, together with the duality lemma 2.1, shows that a terminal segment of $p$ $k$-subsets of $[n]$ in squashed order minimises the size of the shade over all collections of $p$ $k$-sets:

Corollary 2.7. If $\mathcal{B}$ is a collection of $k$-subsets of $[n]$ then

$$
|\nabla \mathcal{B}| \geq\left|\nabla L_{n, k}(|\mathcal{B}|)\right|
$$

A very important consequence of Theorem 2.6 is the fact that if $\mathcal{A}$ is an antichain on $[n]$, then there exists a squashed antichain on $[n]$ with the same parameters as $\mathcal{A}$. 
Theorem 2.8 (Clements [3], Daykin et al. [6]). There exists an antichain on $[n]$ with parameters $p_{0}, \ldots, p_{n}$ if and only if there exists a squashed antichain with the same parameters.

Well-known results by Clements give lower bounds and upper bounds for the size of the new-shadows and new-shades.

Theorem 2.9 (Clements [5]). Let $p \in \boldsymbol{N}$ be such that $p \leq\left(\begin{array}{l}n \\ k\end{array}\right)$. Then

$$
\left|\triangle F_{n, k}(p)\right| \geq\left|\triangle_{N} C_{n, k}(p)\right| \geq\left|\triangle_{N} L_{n, k}(p)\right| .
$$

The dual statement reads as

Corollary 2.10. Let $p \in N$ be such that $p \leq\left(\begin{array}{l}n \\ k\end{array}\right)$. Then

$$
\left|\nabla L_{n, k}(p)\right| \geq\left|\nabla_{N} C_{n, k}(p)\right| \geq\left|\nabla_{N} F_{n, k}(p)\right| .
$$

Note that in Theorem 2.9 and Corollary 2.10, the collection $C_{n, k}(p)$ denotes any collection of $p$ consecutive $k$-subsets of $[n]$ in squashed order.

Theorem 2.11 (Clements [5]). Let $p \in \boldsymbol{N}$ be such that $p \leq \min \left\{\left(\begin{array}{l}n \\ k\end{array}\right),\left(\begin{array}{c}n \\ k+1\end{array}\right)\right\}$. Then

$$
\begin{aligned}
& \left|\triangle F_{n, k}(p)\right| \leq\left|\triangle F_{n, k+1}(p)\right|, \text { and } \\
& \left|\triangle_{N} L_{n, k}(p)\right| \leq\left|\triangle_{N} L_{n, k+1}(p)\right| .
\end{aligned}
$$

\section{The Proof of Theorem 1.5 : Part A}

See Figure 1 for an outline of the proof of Theorem 1.5.

Proposition 3.1. Theorem 1.5 holds for $1 \leq k \leq \frac{n+1}{3}$.

Proof. For $k=p=1$ this is trivial. For $k=1$ and $p \neq\left(\begin{array}{c}n \\ k-1\end{array}\right)$ it follows from Sperner's lemma 2.5 that $|\nabla \mathcal{B}|>2 p$. When $p=\left(\begin{array}{c}n \\ k-1\end{array}\right),|\nabla \mathcal{B}| \geq 2 p$ and $\left|\triangle_{N} \mathcal{A}\right|>0$.

Proposition 3.2. Theorem 1.5 holds for $n \leq 32$.

Proof. By exhaustive computations. 


\section{Part A}

$$
\begin{aligned}
& 1 \leq k \leq \frac{n+1}{3} \\
& \text { Proposition } 3.1 \\
& n \leq 32 \\
& n>32, \frac{n+1}{3}<k \leq \frac{n}{2}, 1 \leq p \leq\left(\begin{array}{l}
n-1 \\
k-2
\end{array}\right) \\
& n>32, \frac{n+1}{3}<k \leq \frac{n}{2},\left(\begin{array}{l}
n-1 \\
k-2
\end{array}\right)<\left(\begin{array}{l}
n-2 \\
k-1
\end{array}\right),\left(\begin{array}{l}
n-1 \\
k-2
\end{array}\right)<p \leq\left(\begin{array}{l}
n-2 \\
k-1
\end{array}\right) \\
& n>32, \frac{n+1}{3}<k \leq \frac{n}{2},\left(\begin{array}{l}
n-1 \\
k-2
\end{array}\right)<\left(\begin{array}{l}
n-2 \\
k-1
\end{array}\right),\left(\begin{array}{l}
n-2 \\
k-1
\end{array}\right)<p \leq\left(\begin{array}{c}
n-1 \\
k
\end{array}\right) \\
& n>32, \frac{n+1}{3}<k \leq \frac{n}{2},\left(\begin{array}{l}
n-1 \\
k-2
\end{array}\right)<\left(\begin{array}{c}
n-2 \\
k-1
\end{array}\right),\left(\begin{array}{c}
n-1 \\
k
\end{array}\right)<p \leq\left(\begin{array}{c}
n \\
k-1
\end{array}\right) \\
& n>32, \frac{n+1}{3}<k \leq \frac{n}{2},\left(\begin{array}{l}
n-1 \\
k-2
\end{array}\right) \geq\left(\begin{array}{l}
n-2 \\
k-1
\end{array}\right) \text {, } \\
& \left(\begin{array}{l}
n-1 \\
k-2
\end{array}\right)<p \leq\left(\begin{array}{c}
n-1 \\
k-2
\end{array}\right)+\left(\begin{array}{c}
n-2 \\
k
\end{array}\right) \\
& n>32, \frac{n+1}{3}<k \leq \frac{n}{2},\left(\begin{array}{l}
n-1 \\
k-2
\end{array}\right) \geq\left(\begin{array}{l}
n-2 \\
k-1
\end{array}\right), \frac{k-1}{n-k}+\frac{n-k-1}{k} \geq 2, \\
& \left(\begin{array}{c}
n-1 \\
k-2
\end{array}\right)+\left(\begin{array}{c}
n-2 \\
k
\end{array}\right)<p \leq\left(\begin{array}{c}
n \\
k-1
\end{array}\right)
\end{aligned}
$$

\section{Part B}

$$
\begin{aligned}
& n>32, \frac{n+1}{3}<k \leq \frac{n}{2},\left(\begin{array}{l}
n-1 \\
k-2
\end{array}\right) \geq\left(\begin{array}{l}
n-2 \\
k-1
\end{array}\right), \frac{k-1}{n-k}+\frac{n-k-1}{k}<2, \\
& \left(\begin{array}{l}
n-1 \\
k-2
\end{array}\right)+\left(\begin{array}{c}
n-2 \\
k
\end{array}\right)<p \leq\left(\begin{array}{c}
n \\
k-1
\end{array}\right)
\end{aligned}
$$

Proposition 4.12

\section{Part C}

$$
\begin{aligned}
& 1 n>32, k>\frac{n}{2}, 1 \leq p \leq\left(\begin{array}{c}
n-1 \\
k
\end{array}\right) \\
& n>32, k>\frac{n}{2},\left(\begin{array}{c}
n-2 \\
k-3
\end{array}\right)>\left(\begin{array}{c}
n-1 \\
k
\end{array}\right),\left(\begin{array}{c}
n-1 \\
k
\end{array}\right)<p \leq\left(\begin{array}{c}
n \\
k+1
\end{array}\right) \\
& n>32, k>\frac{n}{2},\left(\begin{array}{c}
n-2 \\
k-3
\end{array}\right) \leq\left(\begin{array}{c}
n-1 \\
k
\end{array}\right),\left(\begin{array}{c}
n-1 \\
k
\end{array}\right)<p \leq\left(\begin{array}{c}
n-1 \\
k-2
\end{array}\right) \\
& n>32, k>\frac{n}{2},\left(\begin{array}{c}
n-2 \\
k-3
\end{array}\right) \leq\left(\begin{array}{c}
n-1 \\
k
\end{array}\right),\left(\begin{array}{c}
n-1 \\
k-2
\end{array}\right)<p \leq\left(\begin{array}{c}
n \\
k+1
\end{array}\right)
\end{aligned}
$$

Proposition 5.1

Proposition 5.2

Proposition 5.3

Proposition 5.4

Figure 1: Outline of the cases considered in the proof of Theorem 1.5 
All subsequent proofs in this section and Sections 4 and 5 are proofs by induction on $n$. The induction hypothesis is

Induction Hypothesis 3.3 (IH 3.3). Theorem 1.5 holds for all positive integers less than $n$.

In each of the following propositions we show that the collections $L_{n, k+1}(p)$ and $L_{n, k-1}(p)$ satisfy Theorem 1.5. The two collections $L_{n, k+1}(p)$ and $L_{n, k-1}(p)$ are partitioned into $\left\{\mathcal{A}_{1}, \mathcal{A}_{2}, \ldots, \mathcal{A}_{m}\right\}$ and $\left\{\mathcal{B}_{1}, \mathcal{B}_{2}, \ldots, \mathcal{B}_{m}\right\}$ respectively and it is shown that for each $i$, $i=1, \ldots, m,\left|\triangle_{N} \mathcal{A}_{i}\right|+\left|\nabla_{N} \mathcal{B}_{i}\right| \geq 2 \max \left\{\left|\mathcal{A}_{i}\right|,\left|\mathcal{B}_{i}\right|\right\}$ with a strict inequality occurring for at least one value of $i$. Finding appropriate partitions for the collections $L_{n, k+1}(p)$ and $L_{n, k-1}(p)$ is relatively easy except in the case dealt with in Part B of the proof (Proposition 4.12).

Proposition 3.4. Let $n>32$ and $\frac{n+1}{3}<k \leq \frac{n}{2}$. Then Theorem 1.5 holds for $p \leq\left(\begin{array}{l}n-1 \\ k-2\end{array}\right)$.

Proof. Since $k \leq \frac{n}{2}, p \leq\left(\begin{array}{c}n-1 \\ k-2\end{array}\right)<\left(\begin{array}{c}n-1 \\ k\end{array}\right)$. Consequently, $\left|\triangle_{N} L_{n, k+1}(p)\right|+\left|\nabla_{N} L_{n, k-1}(p)\right|=$ $\left|\triangle_{N} L_{n-1, k}(p)\right|+\left|\nabla_{N} L_{n-1, k-2}(p)\right|$ and IH 3.3 applies.

Figure 2 illustrates the proof of Proposition 3.4.

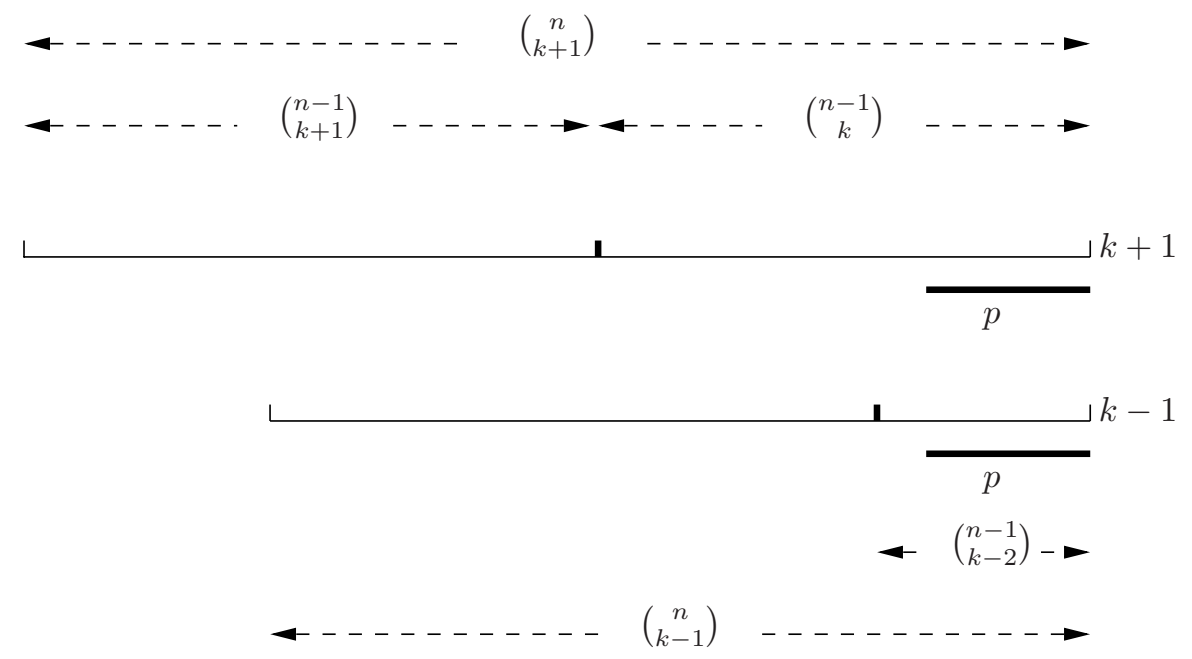

Figure 2: The collections $L_{n, k+1}(p)$ and $L_{n, k-1}(p)$ in Proposition 3.4

The $\left(\begin{array}{c}n \\ k+1\end{array}\right)(k+1)$-sets in squashed order are the $\left(\begin{array}{l}n-1 \\ k+1\end{array}\right)(k+1)$-subsets of $[n-1]$ followed by the $\left(\begin{array}{c}n-1 \\ k\end{array}\right)(k+1)$-subsets of $[n]$ having $n$ as an element. Figure 2 shows this decomposition of the $(k+1)$-sets and a similar decomposition of the $(k-1)$-sets. The collections $L_{n, k+1}(p)$ and $L_{n, k-1}(p)$ are shown by bold lines marked $p$. 
Similar figures are used to illustrate the remaining proofs. The figures are schematic and are thus not drawn to scale.

Proposition 3.5. Let $n>32$ and $\frac{n+1}{3}<k \leq \frac{n}{2}$ with $\left(\begin{array}{l}n-1 \\ k-2\end{array}\right)<\left(\begin{array}{l}n-2 \\ k-1\end{array}\right)$. Then Theorem 1.5 holds for $\left(\begin{array}{l}n-1 \\ k-2\end{array}\right)<p \leq\left(\begin{array}{l}n-2 \\ k-1\end{array}\right)$.

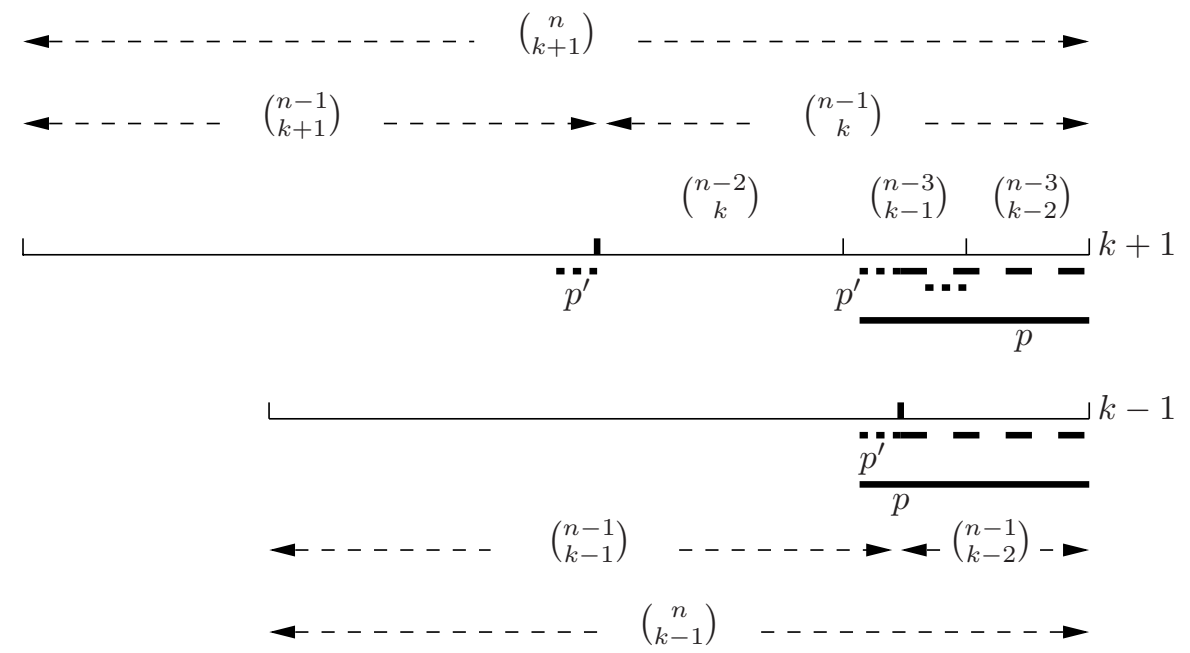

Figure 3: Partitioning $L_{n, k+1}(p)$ and $L_{n, k-1}(p)$ in proving Proposition 3.5

Figure 3 depicts a decomposition of the $(k+1)$-sets relevant to this case. We describe the figure in detail instead of giving a formal proof.

The collection $L_{n, k+1}\left(\left(\begin{array}{c}n-1 \\ k\end{array}\right)\right)$ consists of the $\left(\begin{array}{c}n-2 \\ k\end{array}\right)(k+1)$-sets having $n$ but not $n-1$ as an element, which are followed in squashed order by the $\left(\begin{array}{l}n-2 \\ k-1\end{array}\right)(k+1)$-sets having $n-1$ and $n$ as elements. The collection $L_{n, k+1}\left(\left(\begin{array}{l}n-2 \\ k-1\end{array}\right)\right)$ consists of the $\left(\begin{array}{l}n-3 \\ k-1\end{array}\right)(k+1)$-sets having $n-1$ and $n$ but not $n-2$ as elements, which are followed in squashed order by the $\left(\begin{array}{l}n-3 \\ k-2\end{array}\right)$ $(k+1)$-sets having $n-2, n-1$ and $n$ as elements.

Let $p^{\prime}=p-\left(\begin{array}{l}n-1 \\ k-2\end{array}\right)$; note that $0<p^{\prime} \leq\left(\begin{array}{l}n-3 \\ k-1\end{array}\right)$. Then the first $p^{\prime}$ sets in $L_{n, k-1}(p)$ are isomorphic to $L_{n-1, k-1}\left(p^{\prime}\right)$ and the first $p^{\prime}$ sets in $L_{n, k+1}(p)$ are isomorphic to some collection $C_{n-3, k-1}\left(p^{\prime}\right)$ and we recall that $\left|\triangle_{N} C_{n-3, k-1}\left(p^{\prime}\right)\right| \geq\left|\triangle_{N} L_{n-3, k-1}\left(p^{\prime}\right)\right|$ by Theorem 2.9. Since the collections $L_{n-3, k-1}\left(p^{\prime}\right)$ and $L_{n-1, k+1}\left(p^{\prime}\right)$ are isomorphic, Proposition 3.5 follows from IH 3.3.

Proposition 3.6. Let $n>32$ and $\frac{n+1}{3}<k \leq \frac{n}{2}$ with $\left(\begin{array}{l}n-1 \\ k-2\end{array}\right)<\left(\begin{array}{l}n-2 \\ k-1\end{array}\right)$. Then Theorem 1.5 holds for $\left(\begin{array}{l}n-2 \\ k-1\end{array}\right)<p \leq\left(\begin{array}{c}n-1 \\ k\end{array}\right)$.

Proof. See Figure 4 for an illustration. 


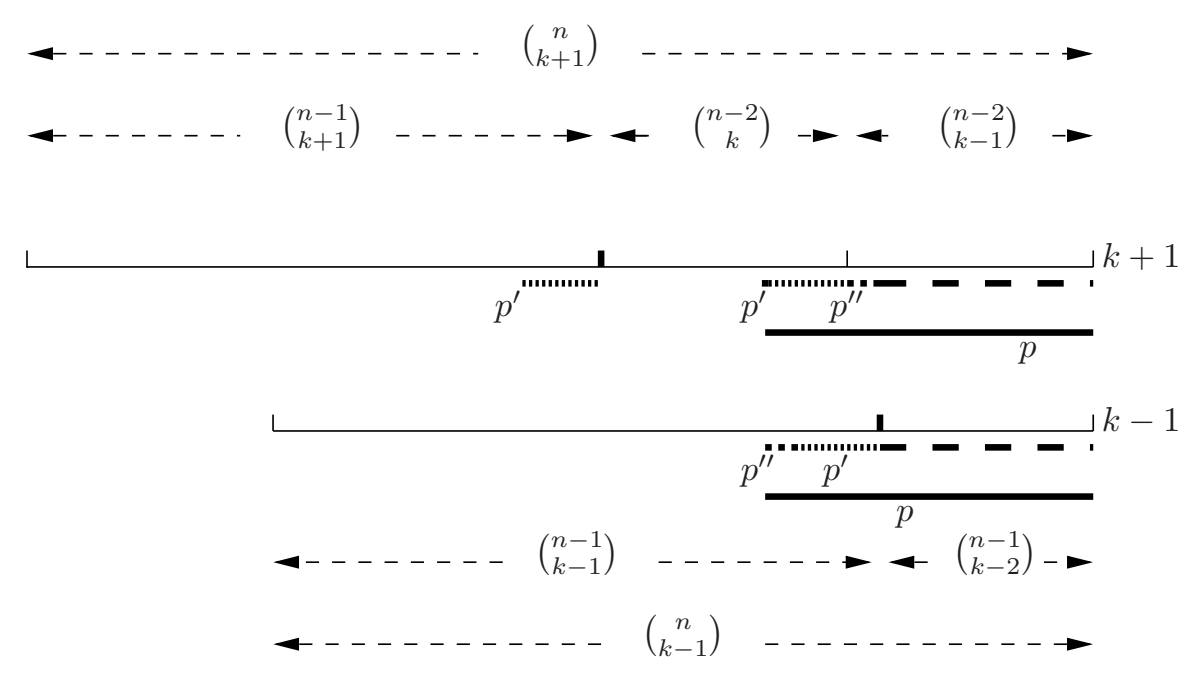

Figure 4: Partitioning $L_{n, k+1}(p)$ and $L_{n, k-1}(p)$ in proving Proposition 3.6

Let $p=p^{\prime}+\left(\begin{array}{c}n-2 \\ k-1\end{array}\right)=p^{\prime \prime}+p^{\prime}+\left(\begin{array}{c}n-1 \\ k-2\end{array}\right)$. Under the current assumptions, $0<p^{\prime} \leq\left(\begin{array}{c}n-2 \\ k\end{array}\right)$. We write $\left|\triangle_{N} L_{n, k+1}(p)\right|=\left|\triangle_{N} L_{n-2, k-1}\left(\left(\begin{array}{c}n-2 \\ k-1\end{array}\right)\right)\right|+\left|\triangle_{N} L_{n-1, k+1}\left(p^{\prime}\right)\right|$ and $\left|\nabla_{N} L_{n, k-1}(p)\right| \geq\left|\nabla_{N} L_{n-1, k-2}\left(\left(\begin{array}{c}n-1 \\ k-2\end{array}\right)\right)\right|+\left|\nabla_{N} L_{n-1, k-1}\left(p^{\prime}\right)\right| . \quad$ Since $\left|\triangle_{N} L_{n-2, k-1}\left(\left(\begin{array}{c}n-2 \\ k-1\end{array}\right)\right)\right|+\left|\nabla_{N} L_{n-1, k-2}\left(\left(\begin{array}{c}n-1 \\ k-2\end{array}\right)\right)\right| \geq 2\left(\begin{array}{c}n-2 \\ k-1\end{array}\right)$ for $k>\frac{n+1}{3}$ the result follows by IH 3.3 .

Proposition 3.7. Let $n>32$ and $\frac{n+1}{3}<k \leq \frac{n}{2}$ with $\left(\begin{array}{c}n-1 \\ k-2\end{array}\right)<\left(\begin{array}{c}n-2 \\ k-1\end{array}\right)$. Then Theorem 1.5 holds for $\left(\begin{array}{c}n-1 \\ k\end{array}\right)<p \leq\left(\begin{array}{c}n \\ k-1\end{array}\right)$.

Proof. Let $p=\left(\begin{array}{c}n-2 \\ k-1\end{array}\right)+p^{\prime}+\left(\begin{array}{c}n-1 \\ k-2\end{array}\right)$. Under the current assumptions, $p^{\prime} \leq\left(\begin{array}{c}n-2 \\ k-2\end{array}\right) \leq\left(\begin{array}{c}n-2 \\ k\end{array}\right)$, $p^{\prime}+\left(\begin{array}{c}n-1 \\ k-2\end{array}\right)>\left(\begin{array}{c}n-2 \\ k\end{array}\right)$, and $\left(\begin{array}{c}n-1 \\ k-2\end{array}\right)<\left(\begin{array}{c}n-2 \\ k\end{array}\right)$. For $n>11$ and $\frac{n+1}{3}<k \leq \frac{n}{2}$, $\left(\begin{array}{c}n-1 \\ k-2\end{array}\right)<\left(\begin{array}{c}n-2 \\ k-1\end{array}\right)$ implies that $\left(\begin{array}{c}n-2 \\ k\end{array}\right)>\left(\begin{array}{c}n-2 \\ k-1\end{array}\right)$. From which it follows that $p^{\prime}>0$.

Using Figure 5 as an illustration, one may write

$$
\begin{aligned}
& \left|\triangle_{N} L_{n, k+1}(p)\right| \\
& \geq\left|\triangle_{N} L_{n-2, k-1}\left(\left(\begin{array}{l}
n-2 \\
k-1
\end{array}\right)\right)\right|+\left|\triangle_{N} L_{n-2, k}\left(\left(\begin{array}{l}
n-1 \\
k-2
\end{array}\right)\right)\right|+\left|\triangle_{N} L_{n-2, k}\left(p^{\prime}\right)\right| \\
& \geq\left|\triangle_{N} L_{n-2, k-1}\left(\left(\begin{array}{l}
n-2 \\
k-1
\end{array}\right)\right)\right|+\left|\triangle_{N} L_{n-2, k-1}\left(\left(\begin{array}{l}
n-1 \\
k-2
\end{array}\right)\right)\right|+\left|\triangle_{N} L_{n-2, k}\left(p^{\prime}\right)\right|
\end{aligned}
$$

(by Theorem 2.11)

$$
=\left|\triangle_{N} L_{n, k+1}\left(\left(\begin{array}{c}
n-2 \\
k-1
\end{array}\right)\right)\right|+\left|\triangle_{N} L_{n-1, k}\left(\left(\begin{array}{l}
n-1 \\
k-2
\end{array}\right)\right)\right|+\left|\triangle_{N} L_{n-2, k}\left(p^{\prime}\right)\right| .
$$




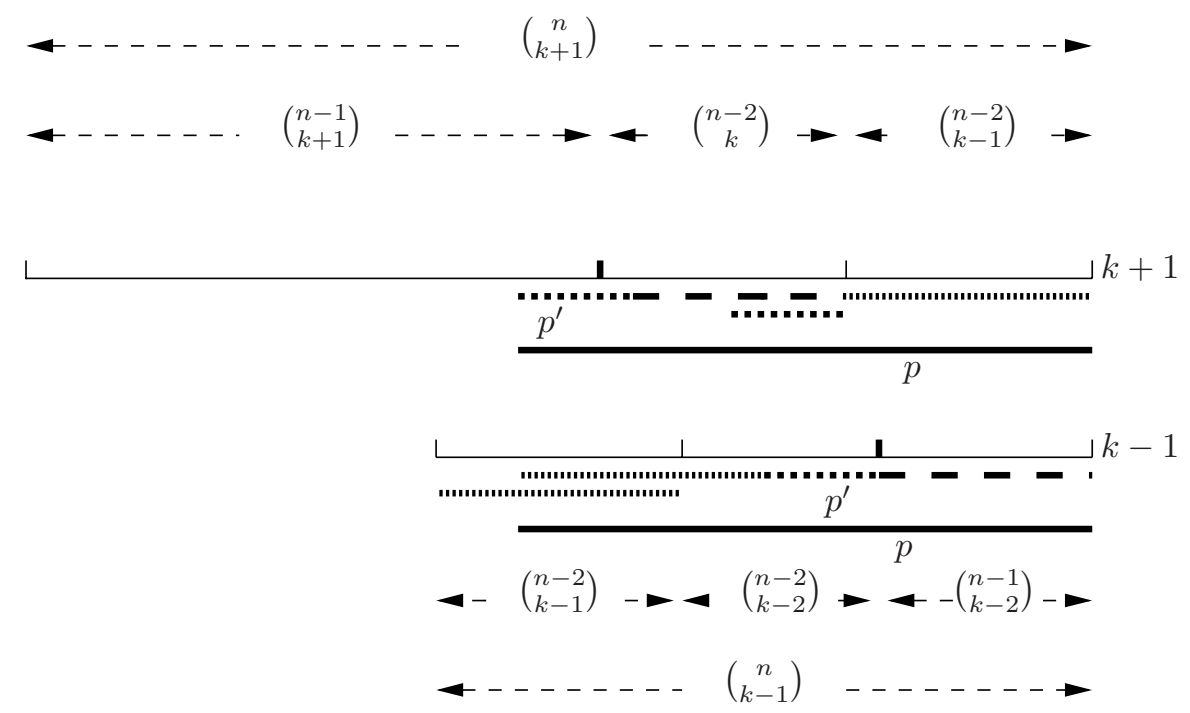

Figure 5: Partitioning of $L_{n, k+1}(p)$ and $L_{n, k-1}(p)$ in proving Proposition 3.7

Applying Corollary 2.10,

$$
\begin{aligned}
& \left|\nabla_{N} L_{n, k-1}(p)\right| \\
& \geq\left|\nabla_{N} L_{n-1, k-2}\left(\left(\begin{array}{l}
n-1 \\
k-2
\end{array}\right)\right)\right|+\left|\nabla_{N} L_{n-2, k-2}\left(p^{\prime}\right)\right|+\left|\nabla_{N} F_{n, k-1}\left(\left(\begin{array}{l}
n-2 \\
k-1
\end{array}\right)\right)\right| .
\end{aligned}
$$

For $n>4$ and $\frac{n+1}{3}<k \leq \frac{n}{2},\left(\begin{array}{l}n-1 \\ k-2\end{array}\right)<\left(\begin{array}{l}n-2 \\ k-1\end{array}\right)$ implies that $\frac{k-1}{n-k}+\frac{n-k-1}{k} \geq 2$, and thus that $\left|\triangle_{N} L_{n, k+1}\left(\left(\begin{array}{l}n-2 \\ k-1\end{array}\right)\right)\right|+\left|\nabla_{N} F_{n, k-1}\left(\left(\begin{array}{l}n-2 \\ k-1\end{array}\right)\right)\right| \geq 2\left(\begin{array}{l}n-2 \\ k-1\end{array}\right)$. Proposition 3.7 follows from this fact and IH 3.3.

Proposition 3.8. Let $n>32$ and $\frac{n+1}{3}<k \leq \frac{n}{2}$ with $\left(\begin{array}{l}n-1 \\ k-2\end{array}\right) \geq\left(\begin{array}{l}n-2 \\ k-1\end{array}\right)$. Then Theorem 1.5 holds for $\left(\begin{array}{l}n-1 \\ k-2\end{array}\right)<p \leq\left(\begin{array}{c}n-1 \\ k-2\end{array}\right)+\left(\begin{array}{c}n-2 \\ k\end{array}\right)$.

Proof. Figure 6 illustrates the proof. Let $p=p^{\prime}+\left(\begin{array}{l}n-1 \\ k-2\end{array}\right)$. Under the current assumptions, we have $0<p^{\prime} \leq\left(\begin{array}{c}n-2 \\ k\end{array}\right)$ and $\left(\begin{array}{l}n-2 \\ k-1\end{array}\right) \leq\left(\begin{array}{c}n-1 \\ k-2\end{array}\right) \leq\left(\begin{array}{c}n-1 \\ k\end{array}\right)$.

Then $\left|\triangle_{N} L_{n, k+1}(p)\right| \geq\left|\triangle_{N} L_{n-1, k}\left(\left(\begin{array}{l}n-1 \\ k-2\end{array}\right)\right)\right|+\left|\triangle_{N} L_{n-1, k+1}\left(p^{\prime}\right)\right|$ and $\left|\nabla_{N} L_{n, k-1}(p)\right|=\left|\nabla_{N} L_{n-1, k-2}\left(\left(\begin{array}{l}n-1 \\ k-2\end{array}\right)\right)\right|+\left|\nabla_{N} L_{n-1, k-1}\left(p^{\prime}\right)\right|$. The result follows from IH 3.3.

Proposition 3.9. Let $n>32$ and $\frac{n+1}{3}<k \leq \frac{n}{2}$ be such that $\left(\begin{array}{l}n-1 \\ k-2\end{array}\right) \geq\left(\begin{array}{l}n-2 \\ k-1\end{array}\right)$ and $\frac{k-1}{n-k}+$ $\frac{n-k-1}{k} \geq 2$. Then Theorem 1.5 holds for $\left(\begin{array}{c}n-1 \\ k-2\end{array}\right)+\left(\begin{array}{c}n-2 \\ k\end{array}\right) \leq p \leq\left(\begin{array}{c}n \\ k-1\end{array}\right)$. 


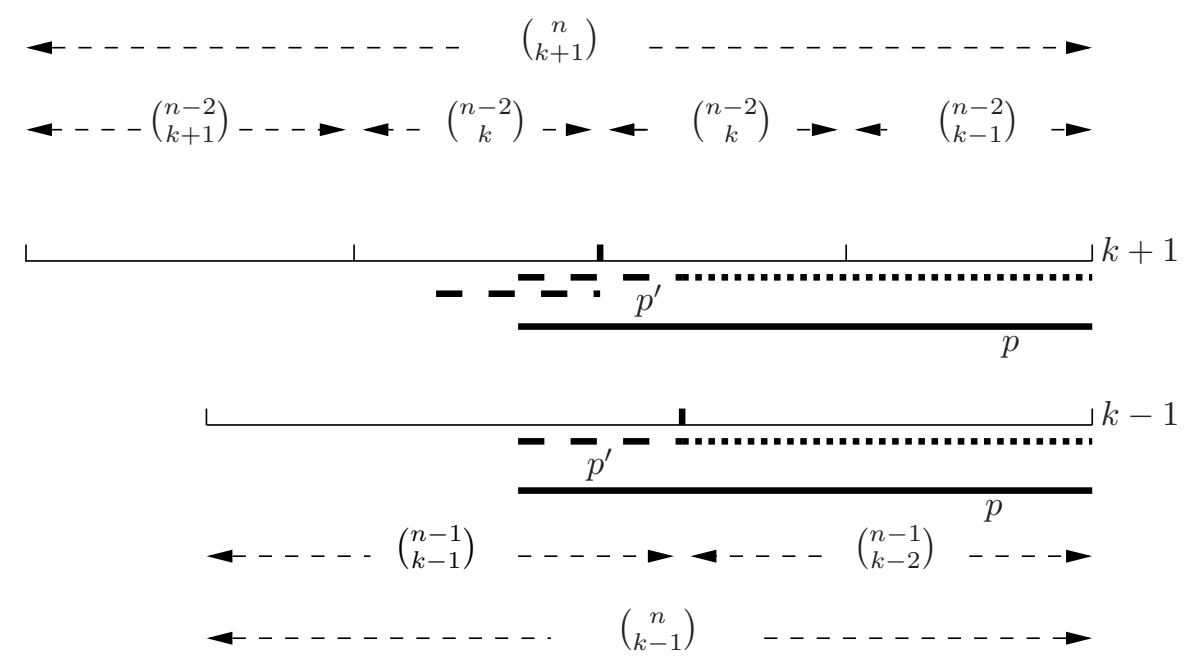

Figure 6: Partitioning of $L_{n, k+1}(p)$ and $L_{n, k-1}(p)$ in proving Proposition 3.8

Proof. Let $p=p^{\prime}+\left(\begin{array}{l}n-2 \\ k-1\end{array}\right)$. Under the current assumptions, $0<p^{\prime} \leq\left(\begin{array}{c}n-2 \\ k\end{array}\right)+\left(\begin{array}{c}n-1 \\ k-2\end{array}\right)$. Then $\left|\triangle_{N} L_{n, k+1}(p)\right| \geq\left|\triangle_{N} L_{n, k+1}\left(\left(\begin{array}{l}n-2 \\ k-1\end{array}\right)\right)\right|+\left|\triangle_{N} L_{n, k+1}\left(p^{\prime}\right)\right|$ and $\left|\nabla_{N} L_{n, k-1}(p)\right| \geq$ $\left|\nabla_{N} L_{n, k-1}\left(p^{\prime}\right)\right|+\left|\nabla_{N} F_{n, k-1}\left(\left(\begin{array}{l}n-2 \\ k-1\end{array}\right)\right)\right|$. We have seen in the proof of Proposition 3.7 that $\left|\triangle_{N} L_{n, k+1}\left(\left(\begin{array}{l}n-2 \\ k-1\end{array}\right)\right)\right|+\left|\nabla_{N} F_{n, k-1}\left(\left(\begin{array}{l}n-2 \\ k-1\end{array}\right)\right)\right| \geq 2\left(\begin{array}{l}n-2 \\ k-1\end{array}\right)$ when $\frac{k-1}{n-k}+\frac{n-k-1}{k} \geq 2$. Proposition 3.9 follows from this fact and from Propositions 3.4 and 3.8 applied to $p^{\prime}$. The proof is illustrated by Figure 7 .

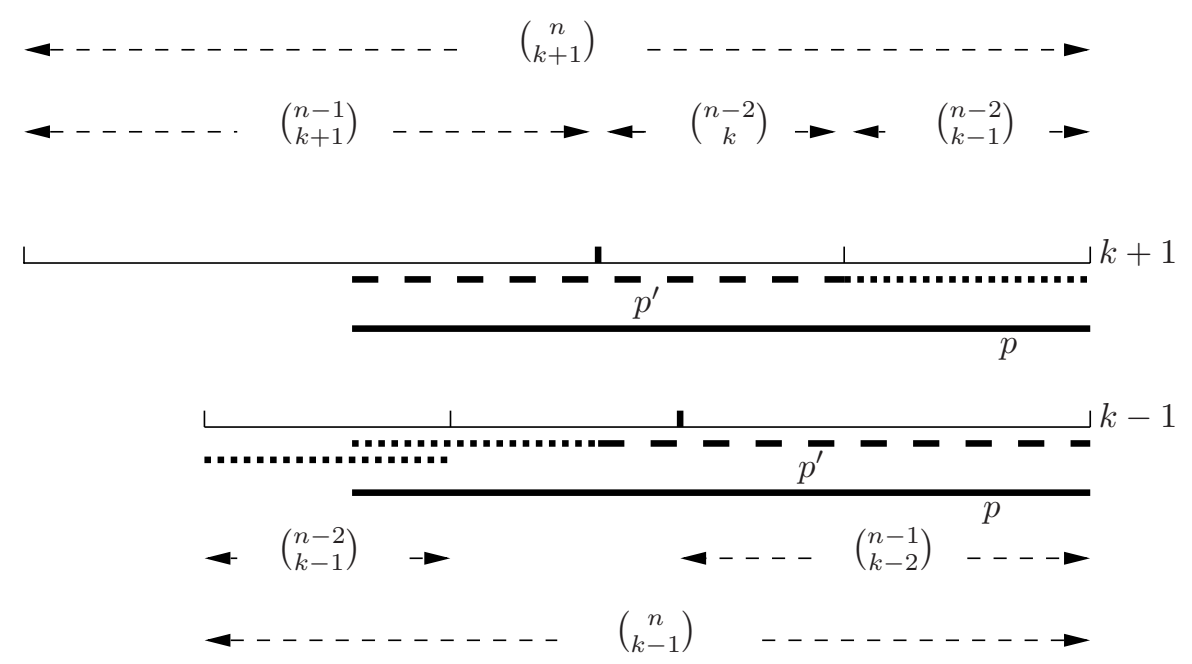

Figure 7: Partitioning of $L_{n, k+1}(p)$ and $L_{n, k-1}(p)$ in proving Proposition 3.9 


\section{The Proof of Theorem 1.5 : Part B}

\subsection{Introduction: Lemma $\mathbf{4 . 2}$}

In order to deal with the case where $n$ and $k$ are such that $n>32, \frac{n+1}{3}<k \leq \frac{n}{2}$, $\left(\begin{array}{l}n-1 \\ k-2\end{array}\right) \geq\left(\begin{array}{l}n-2 \\ k-1\end{array}\right)$, and $\frac{k-1}{n-k}+\frac{n-k-1}{k}<2$, and where $p$ is in the range $\left(\begin{array}{c}n-1 \\ k-2\end{array}\right)+\left(\begin{array}{c}n-2 \\ k\end{array}\right)<p \leq\left(\begin{array}{c}n \\ k-1\end{array}\right)$, we need to digress and prove a technical lemma, Lemma 4.2. Its proof is rather long; in preparation we introduce some terminology and definitions specific to the proof.

All collections are assumed to be collections of sets in squashed order. A collection of consecutive $k$-sets is meant to be a collection of consecutive $k$-sets in squashed order. Whenever we say that a collection $\mathcal{D}$ of $q k$-sets comes before (after) a collection $\mathcal{C}$ of $k$-sets, we mean that $\mathcal{D}$ consists of $q$ consecutive $k$-sets in squashed order that come immediately before (after) the first (last) set in $\mathcal{C}$ in squashed order.

We define $\mathcal{A}$ and $\mathcal{B}$ to be the collections $L_{n, k+1}(p)$ and $L_{n, k-1}(p)$ respectively. For $q \in \boldsymbol{Z}$, $0 \leq q \leq \min \left\{\left(\begin{array}{c}n \\ k+1\end{array}\right),\left(\begin{array}{c}n \\ k-1\end{array}\right)\right\}, 1 \leq k<n$, let $\mathcal{U}$ be a collection of $q(k+1)$-subsets of $[n]$ and $\mathcal{D}$ a collection of $q(k-1)$-subsets of $[n]$. Then the pair $(\mathcal{U}, \mathcal{D})$ is said to have property $P$ if $\left|\triangle_{N} \mathcal{U}\right|+\left|\nabla_{N} \mathcal{D}\right| \geq 2 q$.

Under the current assumptions for $n, k$ and $p,|\mathcal{A}|=p>\left(\begin{array}{c}n-1 \\ k\end{array}\right)$ so that $L_{n, k+1}\left(\left(\begin{array}{c}n-1 \\ k\end{array}\right)\right) \subset \mathcal{A}$.

Lemma 4.1. Let $q$ be given with $0 \leq q \leq\left(\begin{array}{c}n-1 \\ k\end{array}\right)$. Let $\mathcal{U}=F\left(q, L_{n, k+1}\left(\left(\begin{array}{c}n-1 \\ k\end{array}\right)\right)\right)$ and let $\mathcal{D}$ be a collection of $q(k-1)$-sets such that $\left|\nabla_{N} \mathcal{D}\right| \geq \frac{n-k}{k}|\mathcal{D}|$. Then $(\mathcal{U}, \mathcal{D})$ has property $P$.

Proof. Note that $\mathcal{U}$ is isomorphic to $F_{n-1, k}(q)$ and apply Sperner's lemma.

We now state Lemma 4.2 whose proof forms the bulk of this section. This lemma essentially shows that given certain collections of sets $\mathcal{P}_{1} \subseteq \mathcal{A}$ and $\mathcal{P}_{2} \subseteq \mathcal{B}$ with $\left|\mathcal{P}_{1}\right|=\left|\mathcal{P}_{2}\right|$, there exists a way of partitioning them which demonstrates that they have property $\mathrm{P}$ (Apply Lemma 4.1 to see this).

Lemma 4.2. Let $n>32$ and $\frac{n+1}{3}<k \leq \frac{n}{2}$ with $\left(\begin{array}{l}n-1 \\ k-2\end{array}\right) \geq\left(\begin{array}{l}n-2 \\ k-1\end{array}\right)$ and $\frac{k-1}{n-k}+\frac{n-k-1}{k}<2$. Let $p$ be such that $\left(\begin{array}{c}n-1 \\ k-2\end{array}\right)+\left(\begin{array}{c}n-2 \\ k\end{array}\right)<p \leq\left(\begin{array}{c}n \\ k-1\end{array}\right)$.

Let $l \in N$ be such that $0 \leq l \leq k-4$, and let $q_{l}=p-\left(\begin{array}{l}n-2 \\ k-3\end{array}\right)+\sum_{j=1}^{l}\left(\begin{array}{l}n-3-(j-1) \\ k-3-(j-1)\end{array}\right)$. Let $\mathcal{P}_{2}(l)=F\left(q_{l}, \mathcal{B}\right)$.

Then for each $l$, there exists a collection $\mathcal{P}_{1}(l) \subseteq \mathcal{A}$ and partitions $\left\{\mathcal{S}_{1}(l), \mathcal{T}_{1}(l)\right\}$ and $\left\{\mathcal{S}_{2}(l), \mathcal{T}_{2}(l)\right\}$ of $\mathcal{P}_{1}(l)$ and $\mathcal{P}_{2}(l)$ respectively such that

(i) $\mathcal{P}_{1}(l)$ is a collection of $q_{l}$ consecutive $(k+1)$-sets,

(ii) there exists $s \in \boldsymbol{N}$ with

$$
\text { (a) } \mathcal{S}_{1}(l)=F\left(s, L_{n, k+1}\left(\left(\begin{array}{c}
n-1 \\
k
\end{array}\right)\right)\right) \text {, and }
$$




$$
\mathcal{S}_{1}(l)=L\left(s, \mathcal{P}_{1}(l)\right) \text { if } L_{n, k+1}\left(\left(\begin{array}{c}
n-1 \\
k
\end{array}\right)\right) \backslash \mathcal{P}_{1}(l) \neq \emptyset,
$$

(b) $\left|\mathcal{S}_{2}(l)\right|=\left|\mathcal{S}_{1}(l)\right|$ and $\left|\nabla_{N} \mathcal{S}_{2}(l)\right| \geq \frac{n-k}{k}\left|\mathcal{S}_{2}(l)\right|$,

(iii) $\left(\mathcal{T}_{1}(l), \mathcal{T}_{2}(l)\right)$ has property $P$.

To aid readability the notation $\mathcal{P}_{1}, \mathcal{S}_{1}, \mathcal{T}_{1}, \mathcal{P}_{2}, \mathcal{S}_{2}, \mathcal{T}_{2}$ will be used instead of $\mathcal{P}_{1}(l)$, $\mathcal{S}_{1}(l), \mathcal{T}_{1}(l), \mathcal{P}_{2}(l), \mathcal{S}_{2}(l), \mathcal{T}_{2}(l)$. The context will make clear which value of $l$ is under consideration.

Figure 8 pictures the collections $\mathcal{P}_{1}, \mathcal{P}_{2}$ and $\mathcal{S}_{1}$ satisfying the conditions of Lemma 4.2. The collections $\mathcal{A}$ and $\mathcal{B}$ are indicated by a hatched line. Note that the figure assumes that $L_{n, k+1}\left(\left(\begin{array}{c}n-1 \\ k\end{array}\right)\right) \backslash \mathcal{P}_{1} \neq \emptyset$; thus $\mathcal{S}_{1}$ consist of the last $s$ sets of $\mathcal{P}_{1}$.

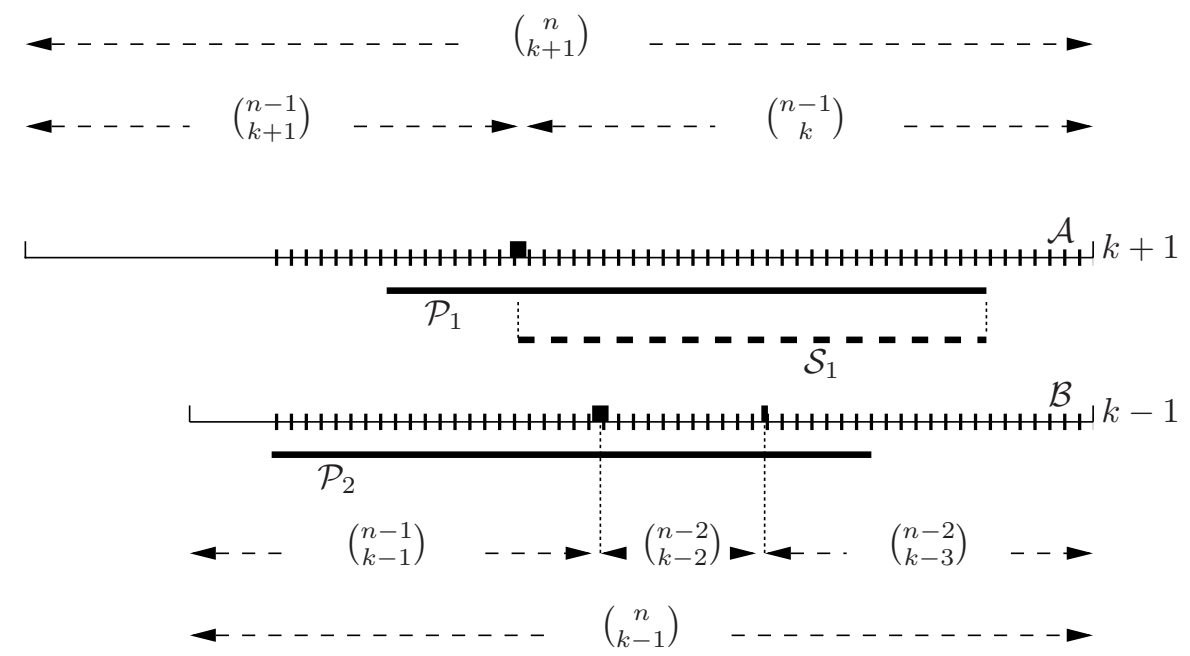

Figure 8: The collections $\mathcal{P}_{1}$ and $\mathcal{P}_{2}$ in Lemma 4.2

The following trivial fact is worth emphasizing: $\mathcal{P}_{1} \subseteq \mathcal{A}$ if and only if $\left|L_{n, k+1}\left(\left(\begin{array}{c}n-1 \\ k\end{array}\right)\right) \backslash \mathcal{P}_{1}\right| \leq p-\left|\mathcal{P}_{2}\right|$.

\subsection{The Proof of Lemma 4.2: Base Case}

Lemma 4.3. Lemma 4.2 holds for $l=0$.

Proof. We consider three cases.

(i) $\left(\begin{array}{l}n-1 \\ k-2\end{array}\right)+\left(\begin{array}{c}n-2 \\ k\end{array}\right)<p \leq\left(\begin{array}{c}n-1 \\ k\end{array}\right)+\left(\begin{array}{c}n-2 \\ k-3\end{array}\right)$ : Figure 9

For $l=0, q_{0}=p-\left(\begin{array}{l}n-2 \\ k-3\end{array}\right)$. Under the present assumptions, $\left(\begin{array}{c}n-2 \\ k-2\end{array}\right)<q_{0} \leq\left(\begin{array}{c}n-1 \\ k\end{array}\right)$. Let $\mathcal{P}_{2}=$ $F\left(q_{0}, \mathcal{B}\right)$ and choose $\mathcal{P}_{1}$ to be the collection $F\left(q_{0}, L_{n, k+1}\left(\left(\begin{array}{c}n-1 \\ k\end{array}\right)\right)\right)$. Let $\mathcal{S}_{1}=\mathcal{P}_{1}$ and $\mathcal{S}_{2}=\mathcal{P}_{2}$ 


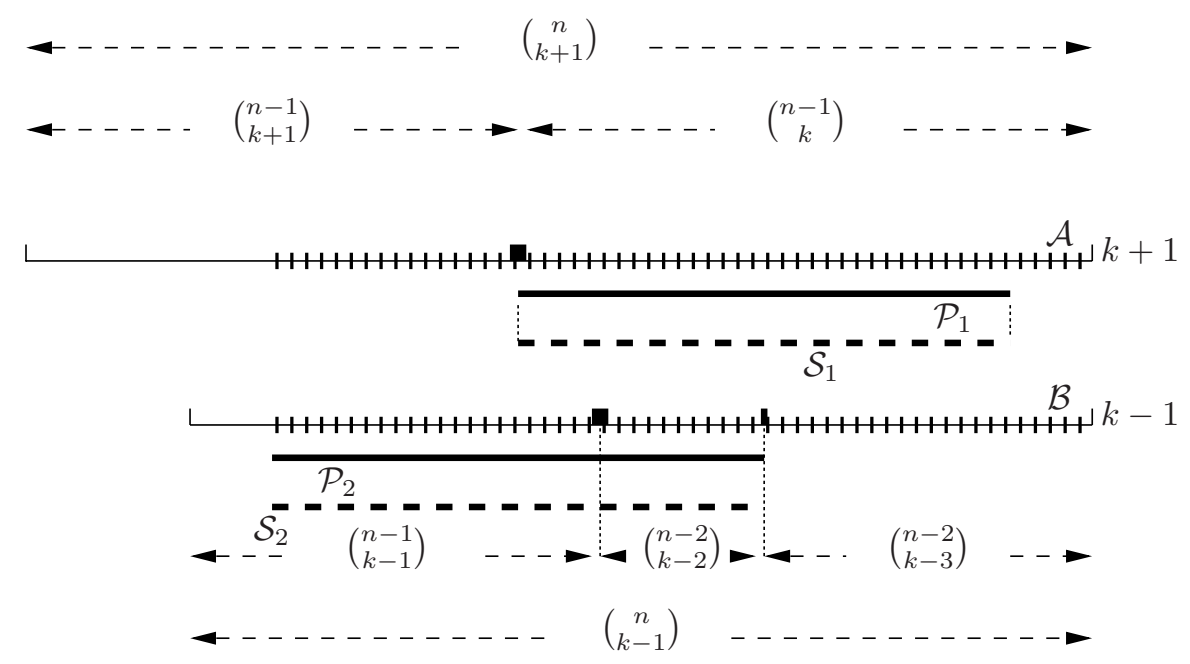

Figure 9: The collections $\mathcal{P}_{1}$ and $\mathcal{P}_{2}$ in proving Lemma 4.3.(i)

so that $\mathcal{T}_{1}=\mathcal{T}_{2}=\emptyset$. It is easy to see that $\left\{L_{n-1, k-1}\left(q_{0}-\left(\begin{array}{l}n-2 \\ k-2\end{array}\right)\right), L_{n-2, k-2}\left(\left(\begin{array}{l}n-2 \\ k-2\end{array}\right)\right) \uplus\{n\}\right\} \quad$ is $\quad$ a $\quad$ partition $\quad$ of $\quad \mathcal{S}_{2}$, so that $\left|\nabla_{N} \mathcal{S}_{2}\right|>\frac{n-k}{k}\left|\mathcal{S}_{2}\right|$ by applying Sperner's lemma.

(ii) $\left(\begin{array}{c}n-1 \\ k\end{array}\right)+\left(\begin{array}{c}n-2 \\ k-3\end{array}\right)<p \leq\left(\begin{array}{c}n \\ k-1\end{array}\right)$ and $\left(\begin{array}{c}n-2 \\ k\end{array}\right)-\left(\begin{array}{c}n-2 \\ k-2\end{array}\right) \leq\left(\begin{array}{c}n-2 \\ k-3\end{array}\right)$ : Figure 10

Here $q_{0}=p-\left(\begin{array}{l}n-2 \\ k-3\end{array}\right)>\left(\begin{array}{l}n-1 \\ k-1\end{array}\right)$. Let $\mathcal{P}_{2}=F\left(q_{0}, \mathcal{B}\right)$ and let $\left\{\mathcal{S}_{2}, \mathcal{T}_{2}\right\}$ be a partition of $\mathcal{P}_{2}$ such that $\mathcal{S}_{2}=F\left(\left(\begin{array}{l}n-1 \\ k-1\end{array}\right), \mathcal{P}_{2}\right)$. Choose $\mathcal{P}_{1}$ to be the collection of $q_{0}$ consecutive $(k+1)$-sets partitioned into $\mathcal{S}_{1}=F\left(\left(\begin{array}{c}n-1 \\ k-1\end{array}\right), L_{n, k+1}\left(\left(\begin{array}{c}n-1 \\ k\end{array}\right)\right)\right)$ and $\mathcal{T}_{1}=L\left(q_{0}-\left(\begin{array}{l}n-1 \\ k-1\end{array}\right), F_{n, k+1}\left(\left(\begin{array}{c}n-1 \\ k+1\end{array}\right)\right)\right)$.

As $\left(\begin{array}{c}n-2 \\ k\end{array}\right)-\left(\begin{array}{c}n-2 \\ k-2\end{array}\right) \leq\left(\begin{array}{c}n-2 \\ k-3\end{array}\right)$ by assumption, $\left|L_{n, k+1}\left(\left(\begin{array}{c}n-1 \\ k\end{array}\right)\right) \backslash \mathcal{P}_{1}\right| \leq p-\left|\mathcal{P}_{2}\right|$ so that $\mathcal{P}_{1}$ is indeed a sub-collection of $\mathcal{A}$.

$\mathcal{S}_{2}$ is a collection $C_{n, k-1}\left(\left(\begin{array}{l}n-1 \\ k-1\end{array}\right)\right)$ and so $\left|\nabla_{N} \mathcal{S}_{2}\right| \geq\left|\nabla_{N} F_{n, k-1}\left(\left(\begin{array}{l}n-1 \\ k-1\end{array}\right)\right)\right|>\frac{n-k+1}{k}\left(\begin{array}{l}n-1 \\ k-1\end{array}\right)$ by Sperner's lemma.

Noting that $q_{0}-\left(\begin{array}{l}n-1 \\ k-1\end{array}\right) \leq\left(\begin{array}{l}n-2 \\ k-2\end{array}\right)$ and $q_{0}-\left(\begin{array}{c}n-1 \\ k-1\end{array}\right) \leq\left(\begin{array}{c}n-2 \\ k\end{array}\right)$, one sees that $\mathcal{T}_{2}$ is the collection $L_{n-2, k-2}\left(q_{0}-\left(\begin{array}{l}n-1 \\ k-1\end{array}\right)\right) \uplus\{n\}$. Since $\mathcal{T}_{1}$ is the collection $\left|\triangle_{N} L_{n-2, k}\left(q_{0}-\left(\begin{array}{l}n-1 \\ k-1\end{array}\right)\right)\right|$, it follows from IH 3.3 that $\left(\mathcal{T}_{1}, \mathcal{T}_{2}\right)$ has property $\mathrm{P}$.

(iii) $\left(\begin{array}{c}n-1 \\ k\end{array}\right)+\left(\begin{array}{c}n-2 \\ k-3\end{array}\right)<p \leq\left(\begin{array}{c}n \\ k-1\end{array}\right)$ and $\left(\begin{array}{c}n-2 \\ k\end{array}\right)-\left(\begin{array}{c}n-2 \\ k-2\end{array}\right)>\left(\begin{array}{c}n-2 \\ k-3\end{array}\right)$ : Figure 11

Here $q_{0}=p-\left(\begin{array}{c}n-2 \\ k-3\end{array}\right)>\left(\begin{array}{c}n-1 \\ k\end{array}\right)$. Let $s=\left(\begin{array}{l}n-1 \\ k-1\end{array}\right)+\left(\begin{array}{l}n-3 \\ k-2\end{array}\right)$. Then, when $\frac{k-1}{n-k}+\frac{n-k-1}{k}<2$ and $\left(\begin{array}{c}n-2 \\ k\end{array}\right)-\left(\begin{array}{c}n-2 \\ k-2\end{array}\right)>\left(\begin{array}{c}n-2 \\ k-3\end{array}\right), s \leq\left(\begin{array}{c}n-1 \\ k\end{array}\right)$ for $n>18$. Thus $q_{0}-s>0$.

Let $\mathcal{P}_{2}=F\left(q_{0}, \mathcal{B}\right)$ and let $\left\{\mathcal{S}_{2}, \mathcal{T}_{2}\right\}$ be a partition of $\mathcal{P}_{2}$ such that $\mathcal{S}_{2}=F\left(s, \mathcal{P}_{2}\right)$. Choose $\mathcal{P}_{1}$ to be the collection of $q_{0}$ consecutive $(k+1)$-sets partitioned into $\mathcal{S}_{1}=F\left(s, L_{n, k+1}\left(\left(\begin{array}{c}n-1 \\ k\end{array}\right)\right)\right)$ 


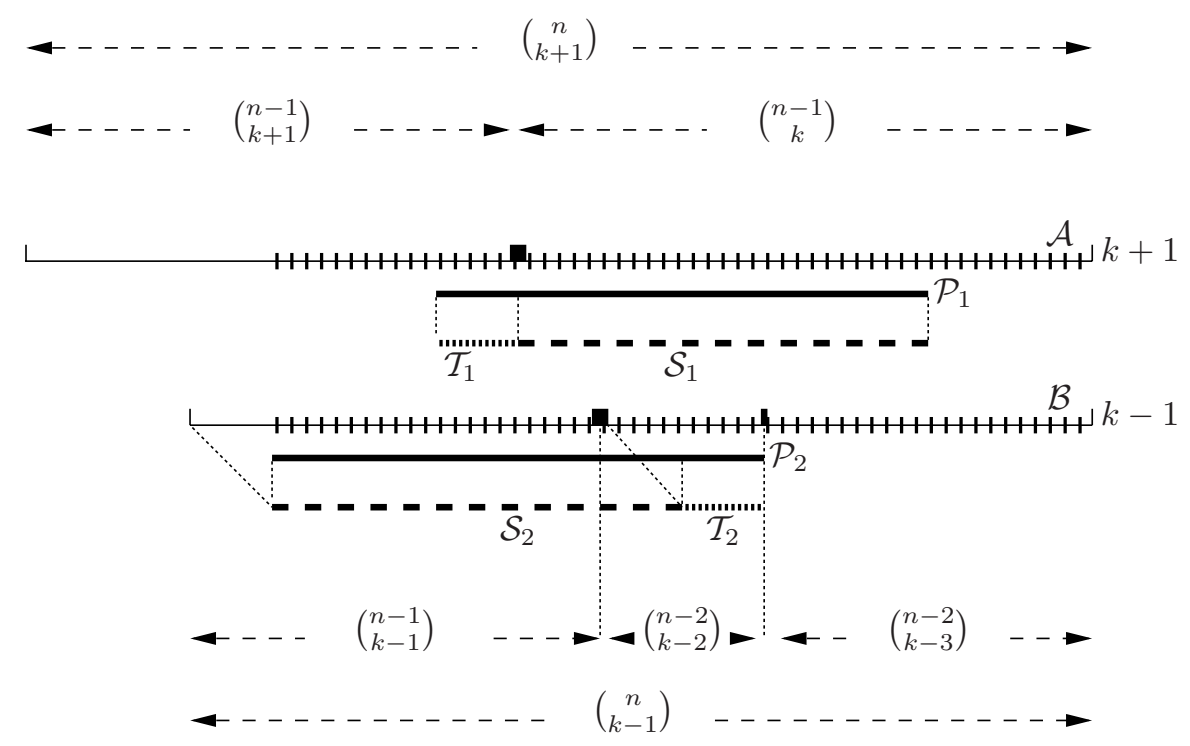

Figure 10: The collections $\mathcal{P}_{1}$ and $\mathcal{P}_{2}$ in proving Lemma 4.3.(ii)
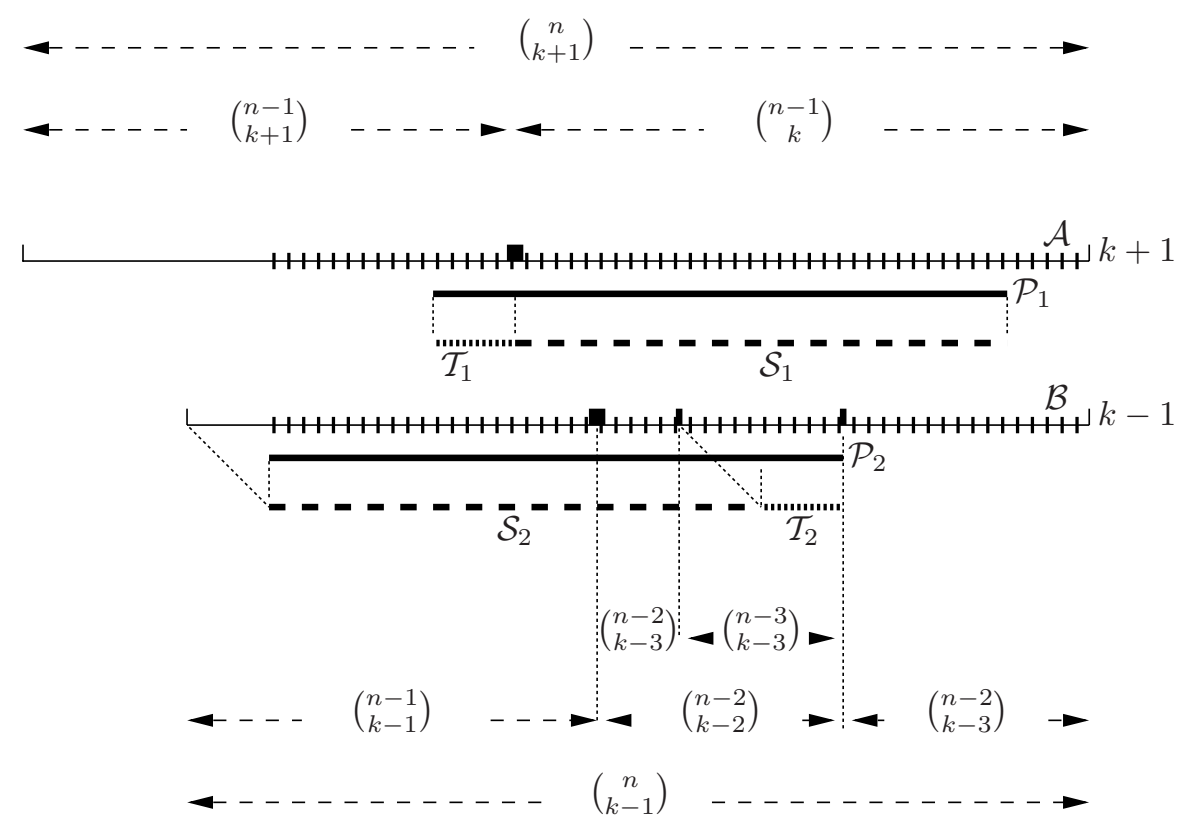

Figure 11: The collections $\mathcal{P}_{1}$ and $\mathcal{P}_{2}$ in proving Lemma 4.3.(iii) 
and $\mathcal{T}_{1}=L\left(q_{0}-s, F_{n, k+1}\left(\left(\begin{array}{l}n-1 \\ k+1\end{array}\right)\right)\right)$.

When $\frac{k-1}{n-k}+\frac{n-k-1}{k}<2$ and $\left(\begin{array}{c}n-2 \\ k\end{array}\right)-\left(\begin{array}{c}n-2 \\ k-2\end{array}\right)>\left(\begin{array}{c}n-2 \\ k-3\end{array}\right)$, it is also the case that $\left(\begin{array}{c}n-2 \\ k\end{array}\right)-\left(\begin{array}{c}n-2 \\ k-2\end{array}\right)-$ $\left(\begin{array}{l}n-3 \\ k-2\end{array}\right) \leq\left(\begin{array}{l}n-2 \\ k-3\end{array}\right)$ for $n>27$. From which it follows that $\left|L_{n, k+1}\left(\left(\begin{array}{c}n-1 \\ k\end{array}\right)\right) \backslash \mathcal{P}_{1}\right| \leq p-\left|\mathcal{P}_{2}\right|$ and so $\mathcal{P}_{1}$ is contained in $\mathcal{A}$.

$\mathcal{S}_{2}$ is a collection $C_{n, k-1}\left(\left(\begin{array}{l}n-1 \\ k-1\end{array}\right)+\left(\begin{array}{l}n-3 \\ k-2\end{array}\right)\right)$. Hence, $\left|\nabla_{N} \mathcal{S}_{2}\right| \geq\left|\nabla_{N} F_{n, k-1}\left(\left(\begin{array}{l}n-1 \\ k-1\end{array}\right)+\left(\begin{array}{l}n-3 \\ k-2\end{array}\right)\right)\right|$ $\geq \frac{n-k}{k}\left|\mathcal{S}_{2}\right|$ by Sperner's lemma.

Under the current assumptions we have $q_{0}-s \leq\left(\begin{array}{l}n-3 \\ k-3\end{array}\right)$ and $q_{0}-s \leq\left(\begin{array}{l}n-3 \\ k-1\end{array}\right)$. It follows that $\mathcal{T}_{2}=L\left(q_{0}-s, \mathcal{P}_{2}\right)$ is isomorphic to $L_{n-3, k-3}\left(q_{0}-s\right)$ and that $\mathcal{T}_{1}$ is isomorphic to $L_{n-3, k-1}\left(q_{0}-s\right)$. Applying IH 3.3 shows that $\left(\mathcal{T}_{1}, \mathcal{I}_{2}\right)$ has property $\mathrm{P}$.

\subsection{The Proof of Lemma 4.2: Inductive Step}

Lemma 4.4. If Lemma 4.2 holds for $l=i$ then Lemma 4.2 holds for $l=i+1$ for all $0 \leq i<k-4$.

The induction hypothesis in the proof is

Induction Hypothesis 4.5 (IH 4.5). Assume that Lemma 4.2 holds for $l=i$.

We denote the collections $\mathcal{P}_{1}(l), \mathcal{S}_{1}(l), \ldots, \mathcal{T}_{2}(l)$ in Lemma 4.2 by $\mathcal{P}_{1}^{\prime}, \mathcal{S}_{1}^{\prime}, \ldots, \mathcal{T}_{2}^{\prime}$ respectively when $l=i$ and by $\mathcal{P}_{1}, \mathcal{S}_{1}, \ldots, \mathcal{T}_{2}$ respectively when $l=i+1$. Note that the dash does not carry any intrinsic meaning and is used for notational convenience only.

In addition to the above notation, let us also define

$q^{\prime}=q_{i}=p-\left(\begin{array}{l}n-2 \\ k-3\end{array}\right)+\sum_{j=1}^{i}\left(\begin{array}{l}n-3-(j-1) \\ k-3-(j-1)\end{array}\right), q=q_{i+1}=p-\left(\begin{array}{l}n-2 \\ k-3\end{array}\right)+\sum_{j=1}^{i+1}\left(\begin{array}{l}n-3-(j-1) \\ k-3-(j-1)\end{array}\right)$, and $m^{\prime}=\left|L_{n, k+1}\left(\left(\begin{array}{c}n-1 \\ k\end{array}\right)\right) \backslash \mathcal{P}_{1}^{\prime}\right|$.

Note that $q-q^{\prime}=\left(\begin{array}{c}n-3-i \\ k-3-i\end{array}\right)$. Let $\mathcal{X}$ be a collection of $(k-1)$-sets such that $\mathcal{X}$ comes after $\mathcal{P}_{2}^{\prime}$ with $\mathcal{P}_{2}=\mathcal{P}_{2}^{\prime} \cup \mathcal{X}$. Thus $|\mathcal{X}|=\left(\begin{array}{c}n-3-i \\ k-3-i\end{array}\right)$.

Let $\mathcal{L}$ and $\mathcal{R}$ be collections of $(k+1)$-sets such that $\mathcal{L}$ comes before $\mathcal{P}_{1}^{\prime}$ and $\mathcal{R}$ comes after $\mathcal{P}_{1}^{\prime}$ with $\mathcal{P}_{1}=\mathcal{L} \cup \mathcal{P}_{1}^{\prime} \cup \mathcal{R}$. Thus $|\mathcal{L}|+|\mathcal{R}|=\left(\begin{array}{l}n-3-i \\ k-3-i\end{array}\right)$.

Figure 12 illustrates the collections $\mathcal{P}_{1}^{\prime}, \mathcal{P}_{2}^{\prime}, \mathcal{L}, \mathcal{R}, \mathcal{X}, \mathcal{P}_{1}$, and $\mathcal{P}_{2}$. Note that it is assumed that $m^{\prime}>0$ when drawing Figure 12 .

Before proceeding, let us observe that:

- $p-\left|\mathcal{P}_{2}^{\prime}\right|=\left(\begin{array}{c}n-2-i \\ k-3-i\end{array}\right)$,

- $\mathcal{L}$ is isomorphic to some collection $C_{n-1, k+1}(|\mathcal{L}|)$,

- $\mathcal{R}$ is isomorphic to some collection $C_{n-1, k}(|\mathcal{R}|)$,

- $\mathcal{X}$ is isomorphic to $L_{n-3-i, k-3-i}\left(\left(\begin{array}{c}n-3-i \\ k-3-i\end{array}\right)\right)$, 


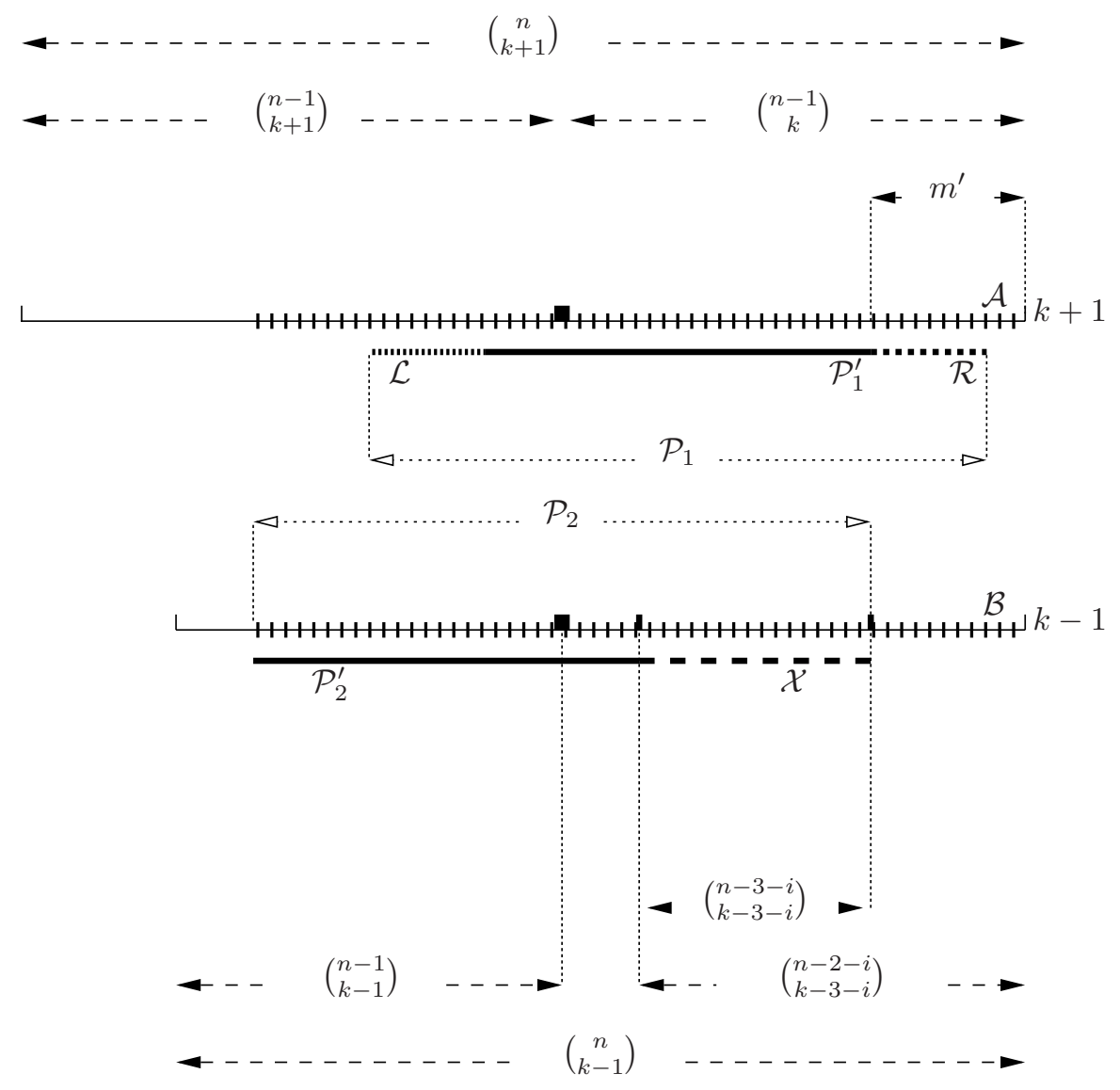

Figure 12: The collections $\mathcal{P}_{1}^{\prime}, \mathcal{P}_{2}^{\prime}, \mathcal{L}, \mathcal{R}, \mathcal{X}, \mathcal{P}_{1}$, and $\mathcal{P}_{2}$.

- $\mathcal{P}_{2}$ is a collection of $q$ consecutive $(k-1)$-sets,

- $\mathcal{P}_{1}$ is a collection of $q$ consecutive $(k+1)$-sets,

- $m^{\prime} \leq p-\left|\mathcal{P}_{2}^{\prime}\right|$ and $|\mathcal{R}| \leq m^{\prime}$.

We now state four lemmas which summarize the basic results required to complete the proof of Lemma 4.4 .

Lemma 4.6. Assume that one of the following conditions holds:

(a) $|\mathcal{R}|=\left(\begin{array}{c}n-3-i \\ k-3-i\end{array}\right)$, or

(b) $m^{\prime} \leq\left(\begin{array}{l}n-3-i \\ k-4-i\end{array}\right)$ and $|\mathcal{R}|=0$, or

(c) $m^{\prime}<\left(\begin{array}{l}n-3-i \\ k-3-i\end{array}\right)$ and $|\mathcal{R}|=\left(\begin{array}{c}n-4-i \\ k-3-i\end{array}\right)$, or

(d) $m^{\prime}<\left(\begin{array}{l}n-4-i \\ k-3-i\end{array}\right)$ and $|\mathcal{R}|=\left(\begin{array}{c}n-5-i \\ k-3-i\end{array}\right)$, or

(e) $m^{\prime}<\left(\begin{array}{l}n-5-i \\ k-3-i\end{array}\right)$ and $|\mathcal{R}|=\left(\begin{array}{c}n-6-i \\ k-3-i\end{array}\right)$. 
Then $\mathcal{P}_{1} \subseteq \mathcal{A}$.

Proof. This is equivalent to showing that in each case, $m^{\prime}-|\mathcal{R}| \leq p-\left|\mathcal{P}_{2}\right|$. In Case (a), this is trivially true. For Case (b), $m^{\prime}-|\mathcal{R}| \leq\left(\begin{array}{l}n-3-i \\ k-4-i\end{array}\right)$. For Cases (c), (d), and (e), $m^{\prime}-|\mathcal{R}|<\left(\begin{array}{c}n-4-i-j \\ k-4-i\end{array}\right)<\left(\begin{array}{c}n-3-i \\ k-4-i\end{array}\right)$ with $j=0,1,2$. Note that $p-\left|\mathcal{P}_{2}\right|=p-\left|\mathcal{P}_{2}^{\prime}\right|-|\mathcal{X}|=$ $\left(\begin{array}{l}n-3-i \\ k-4-i\end{array}\right)$. The result follows.

Lemma 4.7. Let $\mathcal{D}$ be some collection of consecutive $(k-1)$-sets and assume that one of the following conditions holds:

(a) $\mathcal{D}$ is isomorphic to the collection $L_{n-3-i, k-3-i}(|\mathcal{D}|)$, or

(b) $\mathcal{D}$ is isomorphic to the collection $L_{n-4-i, k-3-i}(|\mathcal{D}|)$, or

(c) $\mathcal{D}$ is isomorphic to the collection $L_{n-5-i, k-3-i}(|\mathcal{D}|)$, or

(d) $\mathcal{D}$ is isomorphic to the collection $L_{n-6-i, k-3-i}(|\mathcal{D}|)$ and $\left(\begin{array}{l}n-3-i \\ k-4-i\end{array}\right)<\left(\begin{array}{l}n-5-i \\ k-3-i\end{array}\right)$.

Then $\left|\nabla_{N} \mathcal{D}\right| \geq \frac{n-k}{k}|\mathcal{D}|$.

Proof. In each case, $\mathcal{D}$ is isomorphic to the collection $L_{n-3-i-j, k-3-i}(|\mathcal{D}|)$ for $j=0,1,2,3$. Then $\left|\nabla_{N} \mathcal{D}\right| \geq \frac{n-k-j}{k-2-i}|\mathcal{D}|$ by Sperner's lemma. For $j=0,1,2, \frac{n-k-j}{k-2-i} \geq \frac{n-k}{k}$ since $k \leq \frac{n}{2}$. When $j=3$, the additional condition $\left(\begin{array}{c}n-3-i \\ k-4-i\end{array}\right)<\left(\begin{array}{l}n-5-i \\ k-3-i\end{array}\right)$ applies, in which case $\frac{n-k-3}{k-2-i} \geq \frac{n-k}{k}$ for $n>22$.

Lemma 4.8. Let $\mathcal{U}$ and $\mathcal{D}$ be collections of consecutive $(k+1)$-sets and $(k-1)$-sets respectively. Assume that one of the following conditions holds:

(a) $\mathcal{U}$ is isomorphic to a collection $C_{n-1, k+1}\left(\left(\begin{array}{l}n-3-i \\ k-3-i\end{array}\right)\right)$ and $\mathcal{D}$ is isomorphic to the collection $L_{n-3-i, k-3-i}\left(\left(\begin{array}{c}n-3-i \\ k-3-i\end{array}\right)\right)$, or

(b) $\mathcal{U}$ is isomorphic to a collection $C_{n-1, k+1}\left(\left(\begin{array}{c}n-4-i \\ k-4-i\end{array}\right)\right)$ and $\mathcal{D}$ is isomorphic to the collection $L_{n-4-i, k-4-i}\left(\left(\begin{array}{c}n-4-i \\ k-4-i\end{array}\right)\right)$, or

(c) $\mathcal{U}$ is isomorphic to a collection $C_{n-1, k+1}\left(\left(\begin{array}{l}n-5-i \\ k-4-i\end{array}\right)\right)$ and $\mathcal{D}$ is isomorphic to the collection $L_{n-5-i, k-4-i}\left(\left(\begin{array}{c}n-5-i \\ k-4-i\end{array}\right)\right)$, and $\left(\begin{array}{l}n-3-i \\ k-4-i\end{array}\right)<\left(\begin{array}{c}n-4-i \\ k-3-i\end{array}\right)$, or

(d) $\mathcal{U}$ is isomorphic to a collection $C_{n-1, k+1}\left(\left(\begin{array}{l}n-6-i \\ k-4-i\end{array}\right)\right)$ and $\mathcal{D}$ is isomorphic to the collection $L_{n-6-i, k-4-i}\left(\left(\begin{array}{l}n-6-i \\ k-4-i\end{array}\right)\right)$, and $\left(\begin{array}{l}n-3-i \\ k-4-i\end{array}\right)<\left(\begin{array}{l}n-5-i \\ k-3-i\end{array}\right)$.

Then $(\mathcal{U}, \mathcal{D})$ has property $P$.

Proof. In each case we have $\left|\nabla_{N} \mathcal{D}\right|=\left|\nabla_{N} L_{n-3-i-j, k-3-i-l}\left(\left(\begin{array}{c}n-3-i-j \\ k-3-i-l\end{array}\right)\right)\right|$ where either $j=0$ and $l=0$, or $j=1,2,3$ and $l=1$. 
For Cases (a) and (b), since $\left(\begin{array}{c}n-3-i-j \\ k-3-i-l\end{array}\right) \leq\left(\begin{array}{l}n-1 \\ k+1\end{array}\right)$, we have $\left|\triangle_{N} \mathcal{U}\right| \geq$ $\left|\triangle_{N} L_{n-1, k+1}\left(\left(\begin{array}{c}n-3-i-j \\ k-3-i-l\end{array}\right)\right)\right|=\left|\triangle_{N} L_{n-3-i-j, k-1-i-l}\left(\left(\begin{array}{c}n-3-i-j \\ k-3-i-l\end{array}\right)\right)\right|$ where either $j=l=0$, or $j=l=1$. Apply IH 3.3.

For Case (c) note that $\mathcal{U}$ is isomorphic to a collection $C_{n, k+1}\left(\left(\begin{array}{l}n-5-i \\ k-4-i\end{array}\right)\right)$. Then $\left|\triangle_{N} \mathcal{U}\right| \geq$ $\left|\triangle_{N} L_{n, k+1}\left(\left(\begin{array}{l}n-5-i \\ k-4-i\end{array}\right)\right)\right|=\left(\begin{array}{l}n-5-i \\ k-5-i\end{array}\right)$. The condition $\left(\begin{array}{l}n-3-i \\ k-4-i\end{array}\right)<\left(\begin{array}{l}n-4-i \\ k-3-i\end{array}\right)$ implies that $\left(\begin{array}{c}n-5-i \\ k-5-i\end{array}\right)+$ $\left(\begin{array}{l}n-5-i \\ k-3-i\end{array}\right) \geq 2\left(\begin{array}{l}n-5-i \\ k-4-i\end{array}\right)$ when $n>22$.

For Case $(\mathrm{d}),\left|\triangle_{N} \mathcal{U}\right| \geq\left|\triangle_{N} L_{n-1, k+1}\left(\left(\begin{array}{l}n-6-i \\ k-4-i\end{array}\right)\right)\right|=\left(\begin{array}{l}n-6-i \\ k-5-i\end{array}\right)$. The condition $\left(\begin{array}{l}n-3-i \\ k-4-i\end{array}\right)<$ $\left(\begin{array}{l}n-5-i \\ k-3-i\end{array}\right)$ implies that $\left(\begin{array}{l}n-6-i \\ k-5-i\end{array}\right)+\left(\begin{array}{l}n-6-i \\ k-3-i\end{array}\right) \geq 2\left(\begin{array}{l}n-6-i \\ k-4-i\end{array}\right)$ when $n>10$.

The next lemma does not require a proof.

Lemma 4.9. Assume that $\left(\mathcal{X}_{\mathcal{R}}, \mathcal{X}_{\mathcal{L}}\right)$ is a partition of $\mathcal{X}$ with $\mathcal{X}_{\mathcal{R}}$ coming before $\mathcal{X}_{\mathcal{L}}$. Assume that $\left\{\mathcal{S}_{1}, \mathcal{T}_{1}\right\}$ and $\left\{\mathcal{S}_{2}, \mathcal{T}_{2}\right\}$ are partitions of $\mathcal{P}_{1}$ and $\mathcal{P}_{2}$ respectively and assume that one of the following conditions holds:

(a) $\mathcal{S}_{1}=\mathcal{S}_{1}^{\prime} \cup \mathcal{R}$

$\mathcal{T}_{1}=\mathcal{T}_{1}^{\prime} \cup \mathcal{L}$

$\mathcal{S}_{2}=\mathcal{S}_{2}^{\prime} \cup \mathcal{X}_{\mathcal{R}}$

$\mathcal{T}_{2}=\mathcal{T}_{2}^{\prime} \cup \mathcal{X}_{\mathcal{L}}$

with $\left|\nabla_{N} \mathcal{X}_{\mathcal{R}}\right| \geq \frac{n-k}{k}\left|\mathcal{X}_{\mathcal{R}}\right|,|\mathcal{L}|=\left|\mathcal{X}_{\mathcal{L}}\right|$, and $\left(\mathcal{L}, \mathcal{X}_{\mathcal{L}}\right)$ having property $P$,

or

(b) $\mathcal{S}_{1}=\mathcal{S}_{1}^{\prime}$,

$\mathcal{T}_{1}=\mathcal{T}_{1}^{\prime} \cup \mathcal{L} \cup \mathcal{R}$

$\mathcal{S}_{2}=\mathcal{S}_{2}^{\prime}$,

$\mathcal{T}_{2}=\mathcal{T}_{2}^{\prime} \cup \mathcal{X}$

with $\mathcal{S}_{1}^{\prime} \cup \mathcal{R}=L_{n, k+1}\left(\left(\begin{array}{c}n-1 \\ k\end{array}\right)\right)$ and $(\mathcal{L} \cup \mathcal{R}, \mathcal{X})$ having property $P$.

Then the collections $\mathcal{P}_{1}, \mathcal{S}_{1}, \mathcal{T}_{1}, \mathcal{S}_{2}$, and $\mathcal{T}_{2}$ have the following properties:

(i) there exists $s \in \boldsymbol{N}$ with $\mathcal{S}_{1}=F\left(s, L_{n, k+1}\left(\left(\begin{array}{c}n-1 \\ k\end{array}\right)\right)\right)$, and

$$
L\left(s, \mathcal{P}_{1}\right)=\mathcal{S}_{1} \text { if } L_{n, k+1}\left(\left(\begin{array}{c}
n-1 \\
k
\end{array}\right)\right) \backslash \mathcal{P}_{1} \neq \emptyset,
$$

(ii) $\left|\nabla_{N} \mathcal{S}_{2}\right| \geq \frac{n-k}{k}\left|\mathcal{S}_{2}\right|$,

(iii) $\left(\mathcal{T}_{1}, \mathcal{T}_{2}\right)$ has property $P$.

To prove Lemma 4.4 we consider six cases, each case discussing a different value for $m^{\prime}$. In each of the six cases the proof is accompanied by a supporting figure visualising how $\mathcal{P}_{1}$ and $\mathcal{P}_{2}$ are formed. For illustrative purposes the value of $i$ chosen in these figures is $i=0$ and the situation pictured for $i=0$ is that of Lemma 4.3 where $\mathcal{P}_{2}^{\prime}=F\left(p-\left(\begin{array}{l}n-2 \\ k-3\end{array}\right), \mathcal{B}\right)$, 
and $\mathcal{X}$ is the collection of $\left(\begin{array}{l}n-3 \\ k-3\end{array}\right)$ sets that comes after $\mathcal{P}_{2}^{\prime}$. The collections $\mathcal{A}$ and $\mathcal{B}$ are indicated by hatched lines, $\mathcal{P}_{1}^{\prime}$ and $\mathcal{P}_{2}^{\prime}$ are represented by bold lines. The other collections shown are $\mathcal{X}, \mathcal{R}$ and $\mathcal{L}$. None of $\mathcal{S}_{1}, \mathcal{S}_{2}, \mathcal{T}_{1}$ and $\mathcal{T}_{2}$ are shown, so as not to clutter the figures.

1) $\boldsymbol{m}^{\prime} \geq\left(\begin{array}{l}\boldsymbol{n}-\mathbf{3}-\boldsymbol{i} \\ \boldsymbol{k}-\mathbf{3}-\boldsymbol{i}\end{array}\right):$ Figure 13

Let $|\mathcal{R}|=\left(\begin{array}{c}n-3-i \\ k-3-i\end{array}\right),|\mathcal{L}|=0$ and $\mathcal{S}_{1}=\mathcal{S}_{1}^{\prime} \cup \mathcal{R}, \mathcal{S}_{2}=\mathcal{S}_{2}^{\prime} \cup \mathcal{X}, \mathcal{T}_{1}=\mathcal{T}_{1}^{\prime}, \mathcal{T}_{2}=\mathcal{T}_{2}^{\prime}$. Apply Lemmas 4.6, 4.7 and 4.9.

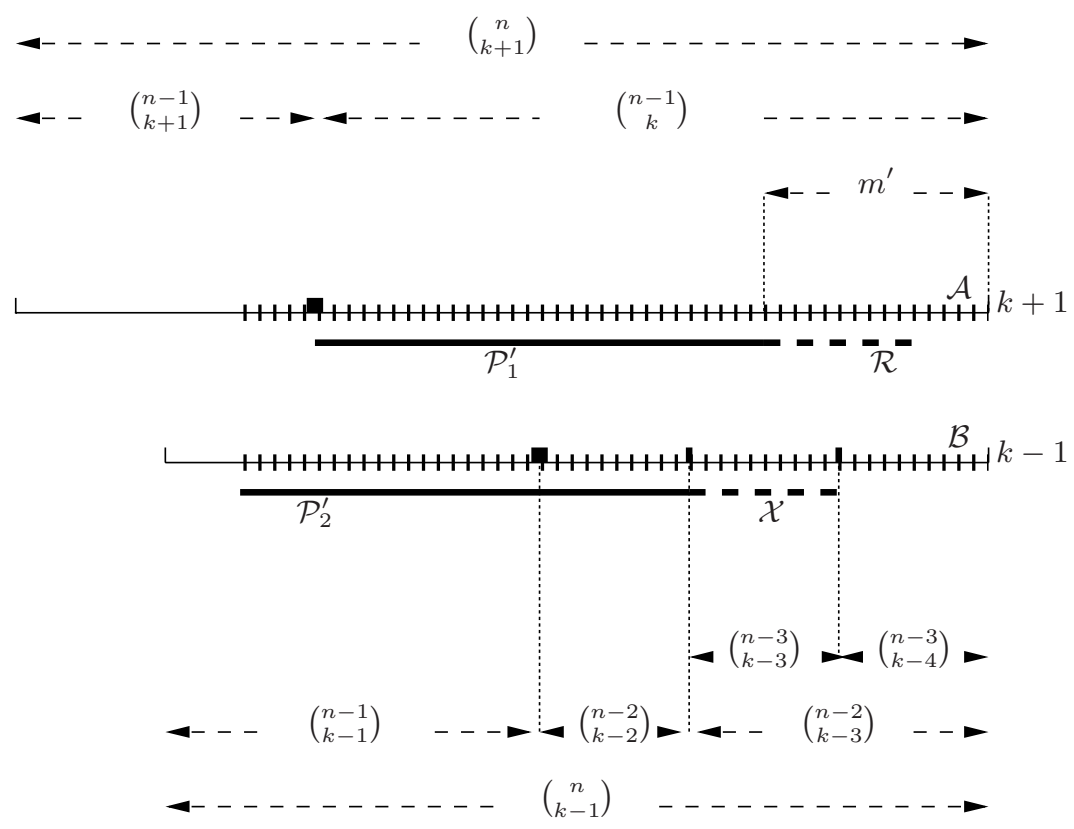

Figure 13: The collections $\mathcal{P}_{1}$ and $\mathcal{P}_{2}$ in case 1) : $m^{\prime} \geq\left(\begin{array}{l}n-3-i \\ k-3-i\end{array}\right)$

2) $\boldsymbol{m}^{\prime} \leq\left(\begin{array}{c}n-3-i \\ k-4-i\end{array}\right) \quad:$ Figure 14

Let $|\mathcal{R}|=0,|\mathcal{L}|=\left(\begin{array}{c}n-3-i \\ k-3-i\end{array}\right)$, and $\mathcal{S}_{1}=\mathcal{S}_{1}^{\prime}, \mathcal{S}_{2}=\mathcal{S}_{2}^{\prime}, \mathcal{T}_{1}=\mathcal{T}_{1}^{\prime} \cup \mathcal{L}, \mathcal{T}_{2}=\mathcal{T}_{2}^{\prime} \cup \mathcal{X}$. Apply Lemmas 4.6, 4.8 and 4.9.

3) $\left(\begin{array}{l}n-3-i \\ k-4-i\end{array}\right)<m^{\prime} \leq\left(\begin{array}{l}n-3-i \\ k-3-i\end{array}\right)$ and $m^{\prime} \geq\left(\begin{array}{l}n-4-i \\ k-3-i\end{array}\right)$ : Figure 15

Let $|\mathcal{R}|=\left(\begin{array}{l}n-4-i \\ k-3-i\end{array}\right),|\mathcal{L}|=\left(\begin{array}{l}n-4-i \\ k-4-i\end{array}\right)$, and partition $\mathcal{X}$ into $\mathcal{X}_{\mathcal{R}}$ and $\mathcal{X}_{\mathcal{L}}$ with $\mathcal{X}_{\mathcal{R}}=F\left(\left(\begin{array}{l}n-4-i \\ k-3-i\end{array}\right), \mathcal{X}\right)$. Then $\left|\mathcal{X}_{\mathcal{L}}\right|=\left(\begin{array}{c}n-4-i \\ k-4-i\end{array}\right) . \quad$ Let $\mathcal{S}_{1}=\mathcal{S}_{1}^{\prime} \cup \mathcal{R}, \quad \mathcal{T}_{1}=\mathcal{T}_{1}^{\prime} \cup \mathcal{L}$, 


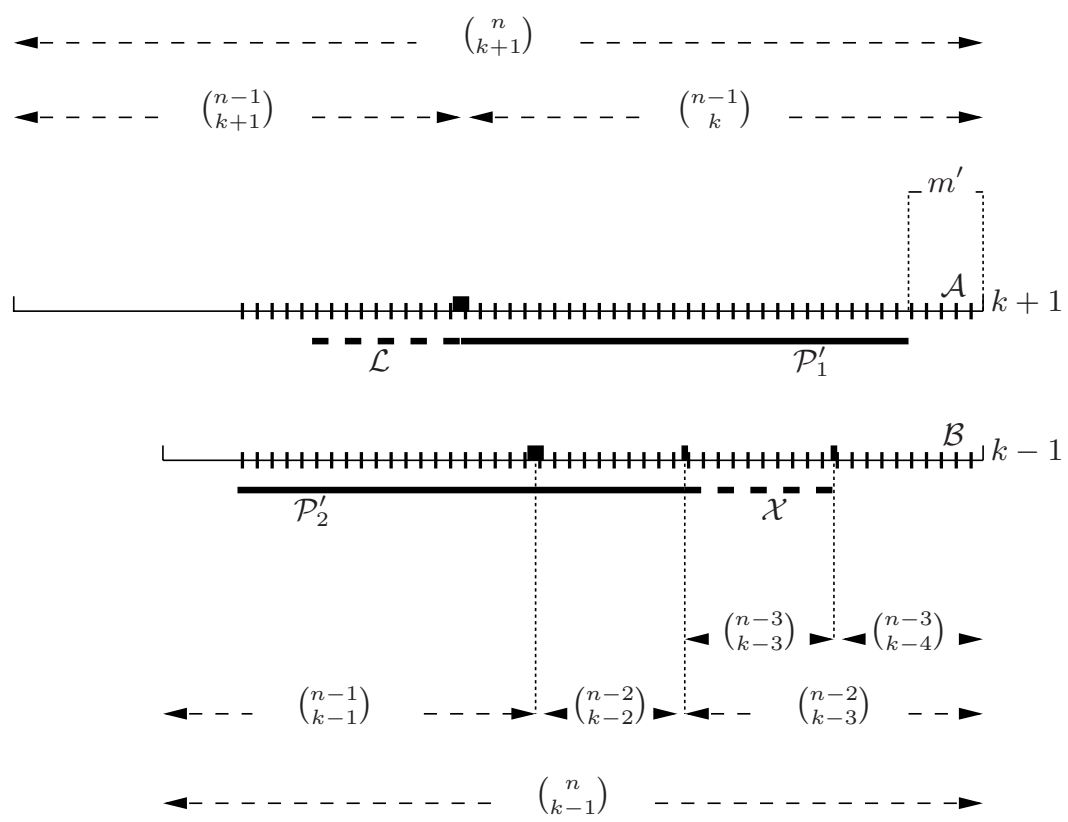

Figure 14: The collections $\mathcal{P}_{1}$ and $\mathcal{P}_{2}$ in case 2$): m^{\prime} \leq\left(\begin{array}{c}n-3-i \\ k-4-i\end{array}\right)$
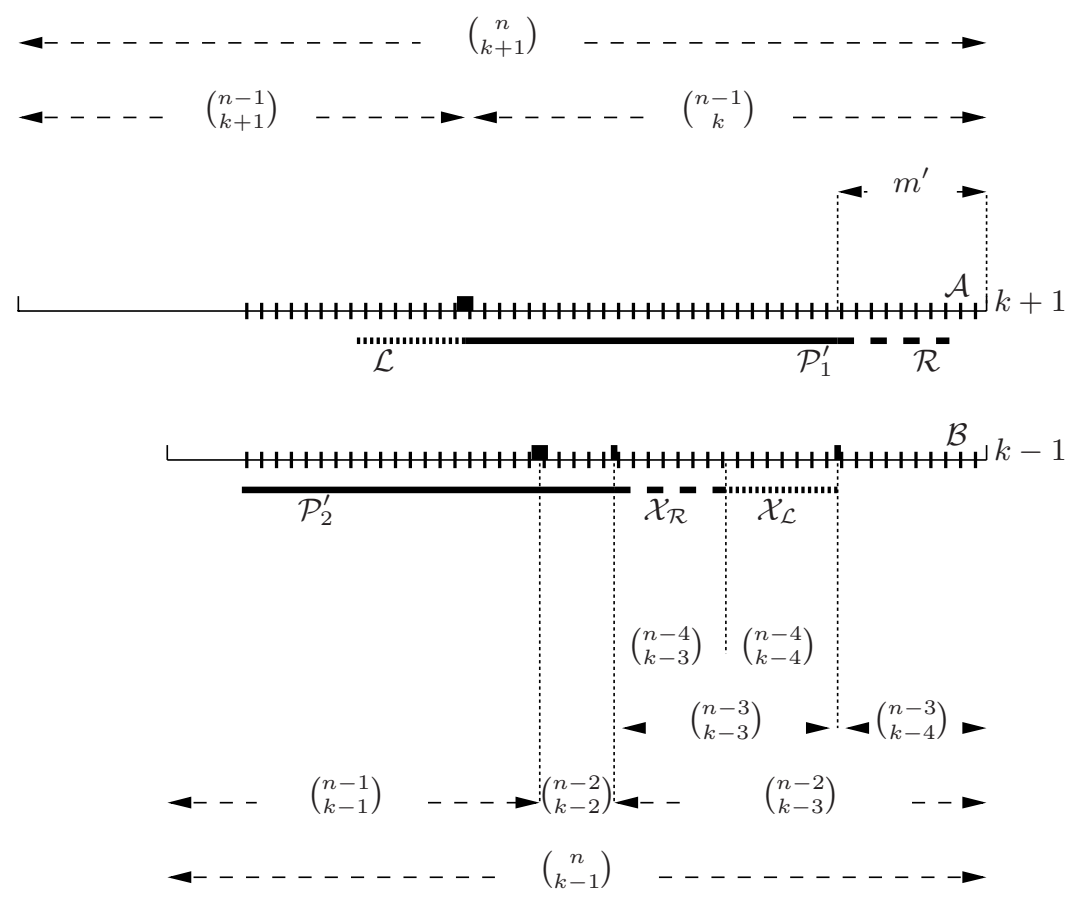

Figure 15: $\mathcal{P}_{1}$ and $\mathcal{P}_{2}$ in case 3): $\left(\begin{array}{l}n-3-i \\ k-4-i\end{array}\right)<m^{\prime}<\left(\begin{array}{l}n-3-i \\ k-3-i\end{array}\right)$ and $m^{\prime} \geq\left(\begin{array}{l}n-4-i \\ k-3-i\end{array}\right)$ 
$\mathcal{S}_{2}=\mathcal{S}_{2}^{\prime} \cup \mathcal{X}_{\mathcal{R}}, \mathcal{T}_{2}=\mathcal{T}_{2}^{\prime} \cup \mathcal{X}_{\mathcal{L}}$ and apply Lemmas $4.6,4.7,4.8$, and 4.9.

4) $\left(\begin{array}{l}n-3-i \\ k-4-i\end{array}\right)<\boldsymbol{m}^{\prime}<\left(\begin{array}{l}n-4-i \\ k-3-i\end{array}\right)$ and $\boldsymbol{m}^{\prime} \geq\left(\begin{array}{l}\boldsymbol{n}-\mathbf{5}-\boldsymbol{i} \\ \boldsymbol{k}-\mathbf{3}-\boldsymbol{i}\end{array}\right)$ : Figure 16

Let $|\mathcal{R}|=\left(\begin{array}{l}n-5-i \\ k-3-i\end{array}\right)$ and $|\mathcal{L}|=\left(\begin{array}{l}n-5-i \\ k-4-i\end{array}\right)+\left(\begin{array}{l}n-4-i \\ k-4-i\end{array}\right)$. Let $\left\{\mathcal{X}_{\mathcal{R}}, \mathcal{X}_{\mathcal{L}}\right\}$ be the partition of $\mathcal{X}$ with $\mathcal{X}_{\mathcal{R}}=F\left(\left(\begin{array}{c}n-5-i \\ k-3-i\end{array}\right), \mathcal{X}\right)$. Thus $\left|\mathcal{X}_{\mathcal{L}}\right|=\left(\begin{array}{l}n-5-i \\ k-4-i\end{array}\right)+\left(\begin{array}{c}n-4-i \\ k-4-i\end{array}\right)$.
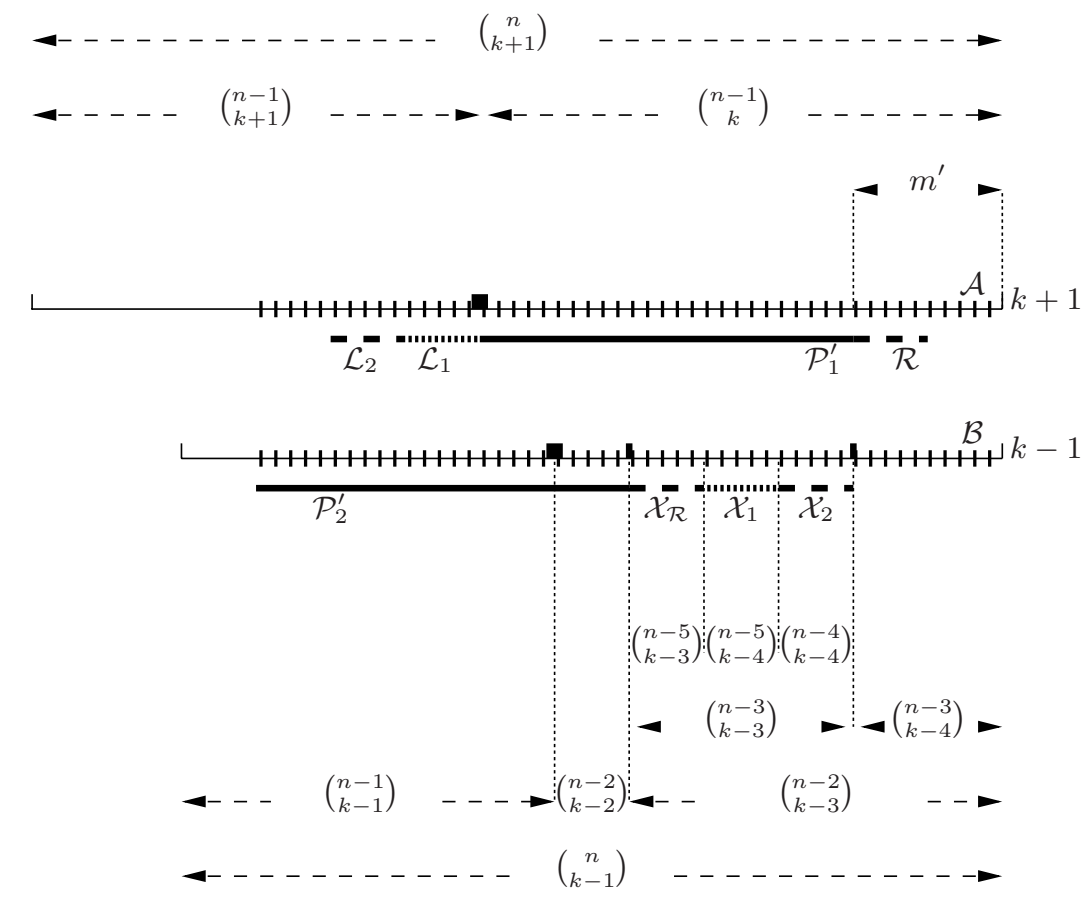

Figure 16: $\mathcal{P}_{1}$ and $\mathcal{P}_{2}$ in case 4): $\left(\begin{array}{l}n-3-i \\ k-4-i\end{array}\right)<m^{\prime}<\left(\begin{array}{l}n-4-i \\ k-3-i\end{array}\right)$ and $m^{\prime} \geq\left(\begin{array}{l}n-5-i \\ k-3-i\end{array}\right)$

To see that $\left(\mathcal{L}, \mathcal{X}_{\mathcal{L}}\right)$ has property $\mathrm{P}$, partition $\mathcal{L}$ and $\mathcal{X}_{\mathcal{L}}$ into $\left\{\mathcal{L}_{1}, \mathcal{L}_{2}\right\}$ and $\left\{\mathcal{X}_{1}, \mathcal{X}_{2}\right\}$ respectively with $\left|\mathcal{L}_{1}\right|=\left(\begin{array}{l}n-5-i \\ k-4-i\end{array}\right),\left|\mathcal{L}_{2}\right|=\left(\begin{array}{l}n-4-i \\ k-4-i\end{array}\right), \mathcal{X}_{1}=F\left(\left(\begin{array}{c}n-5-i \\ k-4-i\end{array}\right), \mathcal{X}_{\mathcal{L}}\right)$, so that $\left|\mathcal{X}_{2}\right|=\left(\begin{array}{c}n-4-i \\ k-4-i\end{array}\right)$. Apply Lemma 4.8 to $\left(\mathcal{L}_{1}, \mathcal{X}_{1}\right)$ and $\left(\mathcal{L}_{2}, \mathcal{X}_{2}\right)$.

Let $\mathcal{S}_{1}=\mathcal{S}_{1}^{\prime} \cup \mathcal{R}, \mathcal{T}_{1}=\mathcal{T}_{1}^{\prime} \cup \mathcal{L}, \mathcal{S}_{2}=\mathcal{S}_{2}^{\prime} \cup \mathcal{X}_{\mathcal{R}}, \mathcal{T}_{2}=\mathcal{T}_{2}^{\prime} \cup \mathcal{X}_{\mathcal{L}}$ and apply Lemmas 4.6, 4.7 and 4.9.

5) $\left(\begin{array}{l}n-3-i \\ k-4-i\end{array}\right)<m^{\prime}<\left(\begin{array}{l}n-5-i \\ k-3-i\end{array}\right)$ and $m^{\prime} \geq\left(\begin{array}{l}n-6-i \\ k-4-i\end{array}\right)$ : Figure 17

This case is similar to Case 4). Let $|\mathcal{R}|=\left(\begin{array}{l}n-6-i \\ k-3-i\end{array}\right),|\mathcal{L}|=\left(\begin{array}{l}n-6-i \\ k-4-i\end{array}\right)+\left(\begin{array}{l}n-5-i \\ k-4-i\end{array}\right)+\left(\begin{array}{l}n-4-i \\ k-4-i\end{array}\right)$. Let $\left\{\mathcal{X}_{\mathcal{R}}, \mathcal{X}_{\mathcal{L}}\right\}$ be the partition of $\mathcal{X}$ with $\mathcal{X}_{\mathcal{R}}=F\left(\left(\begin{array}{l}n-6-i \\ k-3-i\end{array}\right), \mathcal{X}\right)$. Then $\left|\mathcal{X}_{\mathcal{L}}\right|=\left(\begin{array}{c}n-6-i \\ k-4-i\end{array}\right)+$ $\left(\begin{array}{l}n-5-i \\ k-4-i\end{array}\right)+\left(\begin{array}{l}n-4-i \\ k-4-i\end{array}\right)$. 


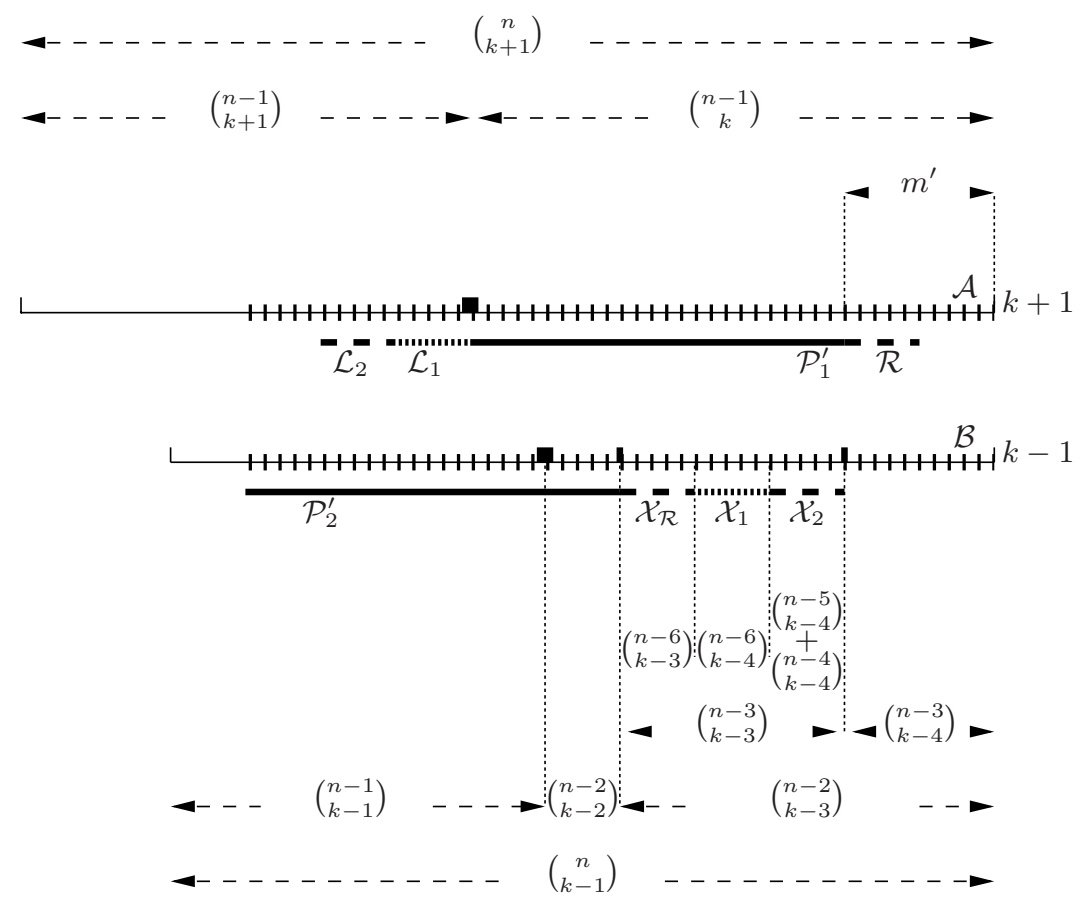

Figure 17: $\mathcal{P}_{1}$ and $\mathcal{P}_{2}$ in case 5): $\left(\begin{array}{l}n-3-i \\ k-4-i\end{array}\right)<m^{\prime}<\left(\begin{array}{l}n-5-i \\ k-3-i\end{array}\right)$ and $m^{\prime} \geq\left(\begin{array}{l}n-6-i \\ k-4-i\end{array}\right)$
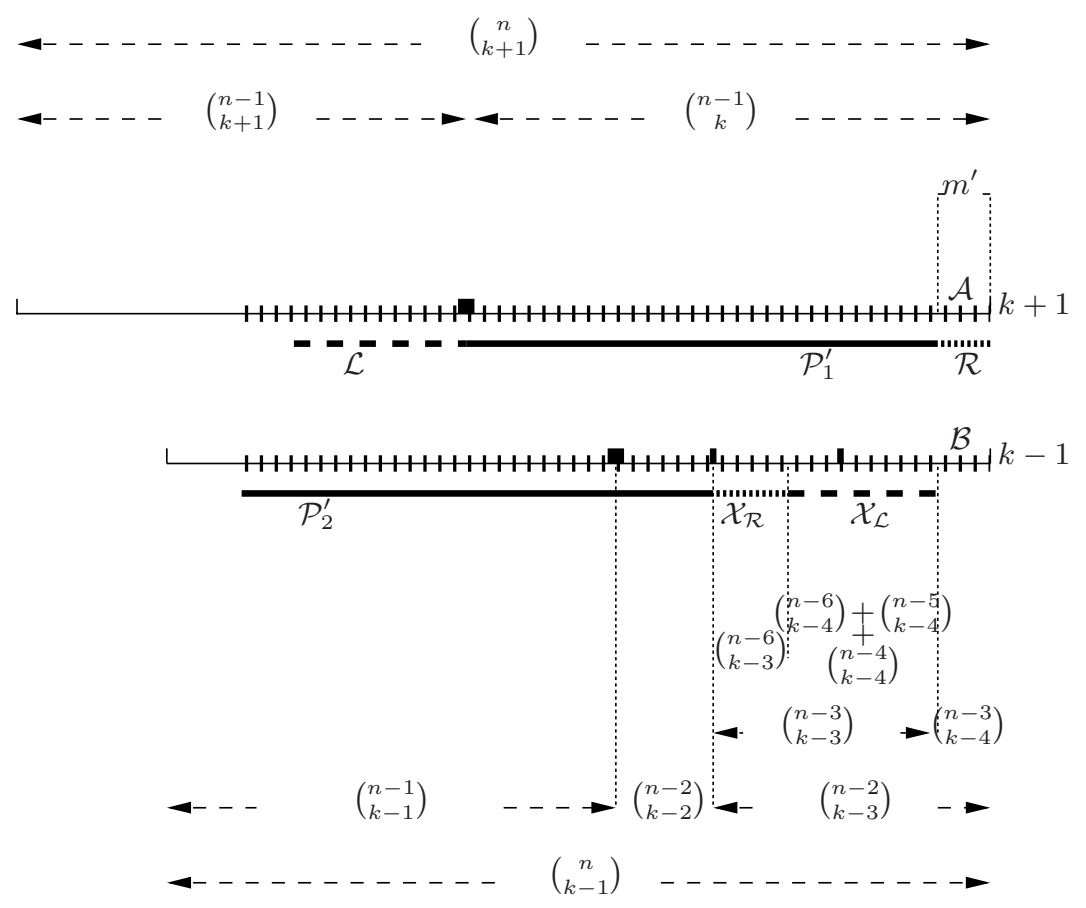

Figure 18: The collections $\mathcal{P}_{1}$ and $\mathcal{P}_{2}$ in case 6) : $\left(\begin{array}{l}n-3-i \\ k-4-i\end{array}\right)<m^{\prime}<\left(\begin{array}{l}n-6-i \\ k-3-i\end{array}\right)$ 
Partition $\mathcal{L}$ and $\mathcal{X}_{\mathcal{L}}$ into $\left\{\mathcal{L}_{1}, \mathcal{L}_{2}\right\}$ and $\left\{\mathcal{X}_{1}, \mathcal{X}_{2}\right\}$ respectively with $\left|\mathcal{L}_{1}\right|=\left(\begin{array}{c}n-6-i \\ k-4-i\end{array}\right),\left|\mathcal{L}_{2}\right|=$ $\left(\begin{array}{l}n-5-i \\ k-4-i\end{array}\right)+\left(\begin{array}{l}n-4-i \\ k-4-i\end{array}\right), \mathcal{X}_{1}=F\left(\left(\begin{array}{l}n-6-i \\ k-4-i\end{array}\right), \mathcal{X}_{\mathcal{L}}\right)$, and apply Lemma 4.8 to see that $\left(\mathcal{L}, \mathcal{X}_{\mathcal{L}}\right)$ has property $\mathrm{P}$.

Let $\mathcal{S}_{1}=\mathcal{S}_{1}^{\prime} \cup \mathcal{R}, \mathcal{T}_{1}=\mathcal{T}_{1}^{\prime} \cup \mathcal{L}, \mathcal{S}_{2}=\mathcal{S}_{2}^{\prime} \cup \mathcal{X}_{\mathcal{R}}, \mathcal{T}_{2}=\mathcal{T}_{2}^{\prime} \cup \mathcal{X}_{\mathcal{L}}$, and apply Lemmas 4.6, 4.7 and 4.9 .

6) $\left(\begin{array}{l}n-3-i \\ k-4-i\end{array}\right)<\boldsymbol{m}^{\prime}<\left(\begin{array}{l}\boldsymbol{n}-6-\boldsymbol{i} \\ \boldsymbol{k}-3-\boldsymbol{i}\end{array}\right)$ : Figure 18

Let $|\mathcal{R}|=m^{\prime},|\mathcal{L}|=\left(\begin{array}{c}n-3-i \\ k-3-i\end{array}\right)-m^{\prime} . \mathcal{X}_{\mathcal{R}}, \mathcal{X}_{\mathcal{L}}$ are as in case 5$)$, and partition $\mathcal{L}$ into $\left\{\mathcal{L}_{1}, \mathcal{L}_{2}\right\}$ such that $\left|\mathcal{L}_{2}\right|=\left|\mathcal{X}_{\mathcal{L}}\right|$. Then $\left(\mathcal{L}_{2}, \mathcal{X}_{\mathcal{L}}\right)$ has property P. For $n>32$ and $\left(\begin{array}{l}n-3-i \\ k-4-i\end{array}\right)<\left(\begin{array}{l}n-6-i \\ k-3-i\end{array}\right)$, we have that $\left(\begin{array}{l}n-6-i \\ k-2-i\end{array}\right) \geq 2\left(\begin{array}{l}n-6-i \\ k-3-i\end{array}\right)$. Then $\left|\nabla_{N} \mathcal{X}_{\mathcal{R}}\right|=\left|\nabla_{N} L_{n-6-i, k-3-i}\left(\left(\begin{array}{c}n-6-i \\ k-3-i\end{array}\right)\right)\right|=\left(\begin{array}{c}n-6-i \\ k-2-i\end{array}\right) \geq 2\left|\mathcal{X}_{\mathcal{R}}\right| . \quad$ This shows that $(\mathcal{L} \cup \mathcal{R}, \mathcal{X})$ has property $\mathrm{P}$.

Let $\mathcal{S}_{1}=\mathcal{S}_{1}^{\prime}, \mathcal{T}_{1}=\mathcal{T}_{1}^{\prime} \cup \mathcal{L} \cup \mathcal{R}, \mathcal{S}_{2}=\mathcal{S}_{2}^{\prime}, \mathcal{T}_{2}=\mathcal{T}_{2}^{\prime} \cup \mathcal{X}$ and apply Lemma 4.9.

\subsection{Proposition 4.12}

At this point, with the proof of Lemma 4.2 behind us, we can state the following:

Lemma 4.10. Let $n>32$ and $\frac{n+1}{3}<k \leq \frac{n}{2}$ with $\left(\begin{array}{l}n-1 \\ k-2\end{array}\right) \geq\left(\begin{array}{l}n-2 \\ k-1\end{array}\right)$ and $\frac{k-1}{n-k}+\frac{n-k-1}{k}<2$. Let $p$ be such that $\left(\begin{array}{c}n-1 \\ k-2\end{array}\right)+\left(\begin{array}{c}n-2 \\ k\end{array}\right)<p \leq\left(\begin{array}{c}n \\ k-1\end{array}\right)$. Let $\mathcal{P}_{2}=F\left(p-\left(\begin{array}{c}n-k+2 \\ 1\end{array}\right), \mathcal{B}\right)$. Then there exists a collection $\mathcal{P}_{1} \subseteq \mathcal{A}$ such that $\left(\mathcal{P}_{1}, \mathcal{P}_{2}\right)$ has property $P$.

Proof. Set $l=k-4$ in Lemma 4.2 .

Lemma 4.11. Let $n>32, k \leq \frac{n}{2},\left(\begin{array}{c}n-1 \\ k-2\end{array}\right)+\left(\begin{array}{c}n-2 \\ k\end{array}\right)<p \leq\left(\begin{array}{c}n \\ k-1\end{array}\right)$, and let $\mathcal{D}=L\left(\left(\begin{array}{c}n-k+2 \\ 1\end{array}\right), \mathcal{B}\right)$. Then $\left|\nabla_{N} \mathcal{D}\right|>2|\mathcal{D}|$.

Proof. Note that $|\mathcal{B}|=p>\left(\begin{array}{c}n-k+2 \\ 1\end{array}\right)$, and that for $n>6, \frac{n-k+1}{2}\left(\begin{array}{c}n-k+2 \\ 1\end{array}\right)>2\left(\begin{array}{c}n-k+2 \\ 1\end{array}\right)$. Let $\mathcal{D}=L\left(\left(\begin{array}{c}n-k+2 \\ 1\end{array}\right), \mathcal{B}\right)$. Then $\mathcal{D}$ is isomorphic to the collection $L_{n-k+2,1}\left(\left(\begin{array}{c}n-k+2 \\ 1\end{array}\right)\right)$. By Sperner's lemma, $\left|\nabla_{N} \mathcal{D}\right|=\left|\nabla L_{n-k+2,1}\left(\left(\begin{array}{c}n-k+2 \\ 1\end{array}\right)\right)\right|=\frac{n-k+1}{2}\left(\begin{array}{c}n-k+2 \\ 1\end{array}\right)>2\left(\begin{array}{c}n-k+2 \\ 1\end{array}\right)$.

Combining Lemmas 4.10 and 4.11 enables us to state:

Proposition 4.12. Let $n>32$ and $\frac{n+1}{3}<k \leq \frac{n}{2}$ with $\left(\begin{array}{l}n-1 \\ k-2\end{array}\right) \geq\left(\begin{array}{l}n-2 \\ k-1\end{array}\right)$ and $\frac{k-1}{n-k}+\frac{n-k-1}{k}<2$. Then Theorem 1.5 holds for $\left(\begin{array}{l}n-1 \\ k-2\end{array}\right)+\left(\begin{array}{c}n-2 \\ k\end{array}\right)<p \leq\left(\begin{array}{c}n \\ k-1\end{array}\right)$. 


\section{The Proof of Theorem 1.5 : Part C}

Proposition 5.1. Let $n>32$ and $k>\frac{n}{2}$. Then Theorem 1.5 holds for $p \leq\left(\begin{array}{c}n-1 \\ k\end{array}\right)$.

Proof. Since $k>\frac{n}{2}, p \leq\left(\begin{array}{c}n-1 \\ k\end{array}\right) \leq\left(\begin{array}{c}n-1 \\ k-2\end{array}\right)$, and the result follows from IH 3.3. See Figure 19 for an illustration.

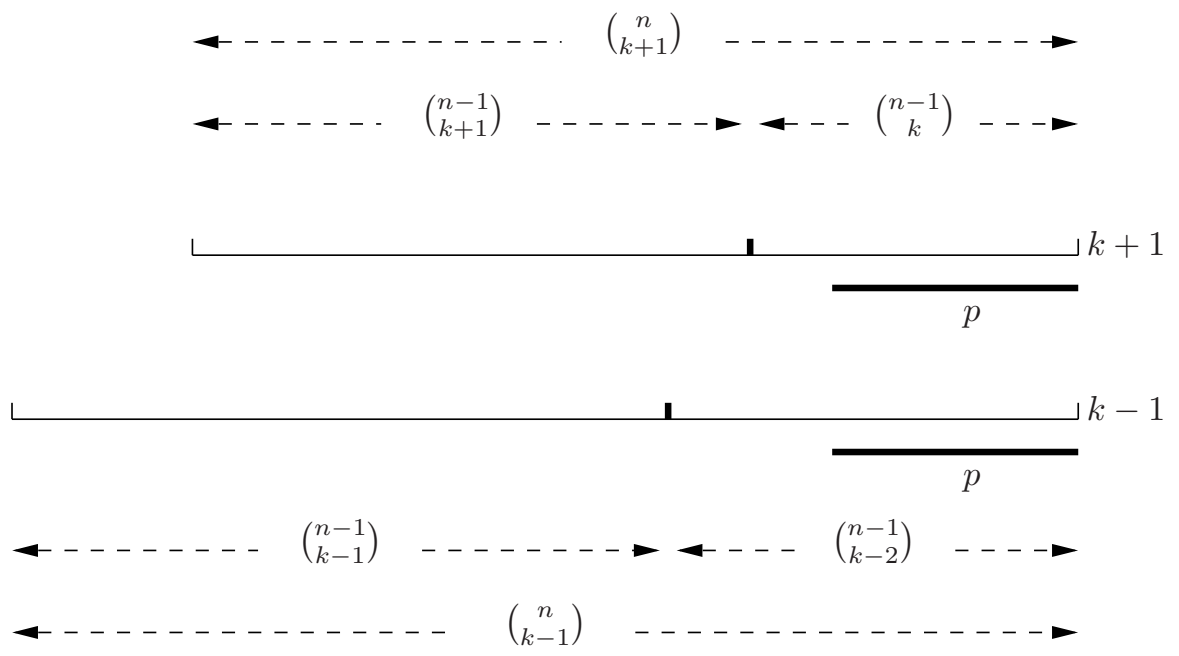

Figure 19: The collections $L_{n, k+1}(p)$ and $L_{n, k-1}(p)$ in Proposition 5.1

Proposition 5.2. Let $n>32$ and $k>\frac{n}{2}$ with $\left(\begin{array}{c}n-2 \\ k-3\end{array}\right)>\left(\begin{array}{c}n-1 \\ k\end{array}\right)$. Then Theorem 1.5 holds for $\left(\begin{array}{c}n-1 \\ k\end{array}\right)<p \leq\left(\begin{array}{c}n \\ k+1\end{array}\right)$.

Proof. Let $p=p^{\prime}+\left(\begin{array}{c}n-1 \\ k\end{array}\right)$. Under the current assumptions, $p<\left(\begin{array}{c}n-1 \\ k-2\end{array}\right)\left(\right.$ as $\left.\left(\begin{array}{c}n \\ k+1\end{array}\right)<\left(\begin{array}{l}n-1 \\ k-2\end{array}\right)\right)$ and $p^{\prime}>0$. Thus

$$
\begin{aligned}
\left|\triangle_{N} L_{n, k+1}(p)\right| & =\left|\triangle_{N} L_{n-1, k}\left(\left(\begin{array}{c}
n-1 \\
k
\end{array}\right)\right)\right|+\left|\triangle_{N} L_{n-1, k+1}\left(p^{\prime}\right)\right| \\
& \geq\left|\triangle_{N} F_{n-1, k}\left(\left(\begin{array}{c}
n-1 \\
k
\end{array}\right)\right)\right|+\left|\triangle_{N} L_{n-1, k}\left(p^{\prime}\right)\right|
\end{aligned}
$$

by Theorem 2.11, and $\left|\nabla_{N} L_{n, k-1}(p)\right| \geq\left|\nabla_{N} L_{n-1, k-2}\left(p^{\prime}\right)\right|+\left|\nabla_{N} F_{n-1, k-2}\left(\left(\begin{array}{c}n-1 \\ k\end{array}\right)\right)\right|$. Proposition 5.2 follows from IH 3.3; see Figure 20 for an illustration. 


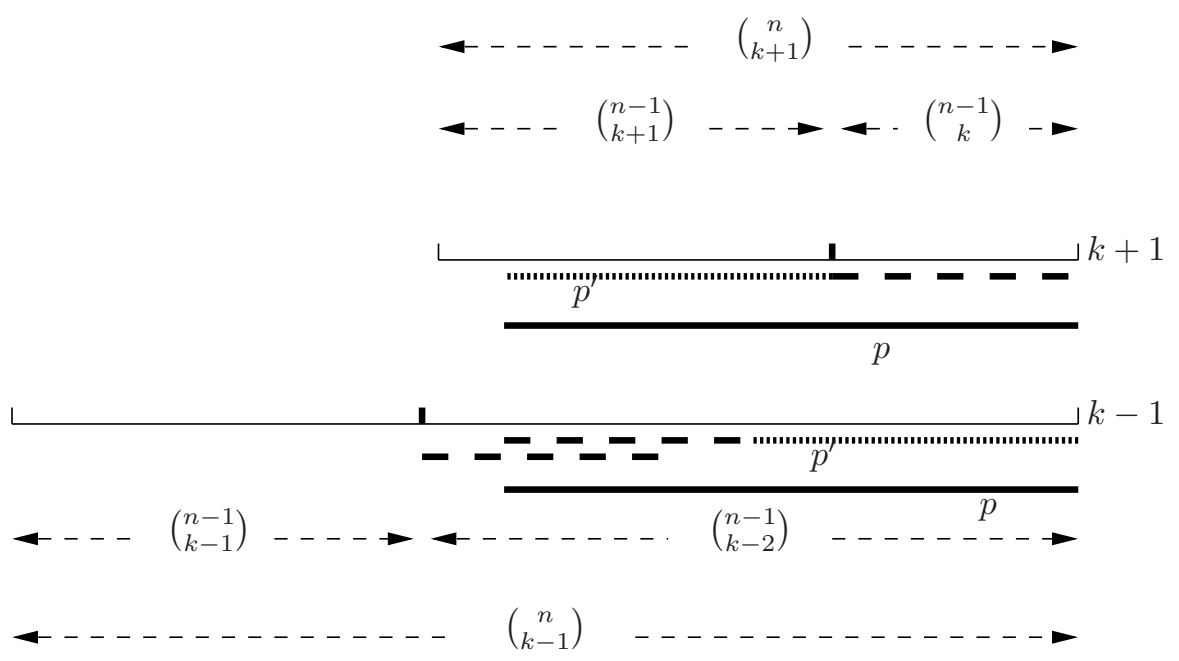

Figure 20: Partitioning of $L_{n, k+1}(p)$ and $L_{n, k-1}(p)$ in proving Proposition 5.2

Proposition 5.3. Let $n>32$ and $k>\frac{n}{2}$ with $\left(\begin{array}{c}n-2 \\ k-3\end{array}\right) \leq\left(\begin{array}{c}n-1 \\ k\end{array}\right)$. Then Theorem 1.5 holds for $\left(\begin{array}{c}n-1 \\ k\end{array}\right)<p \leq\left(\begin{array}{c}n-1 \\ k-2\end{array}\right)$.

Proof. The partitioning of $L_{n, k+1}(p)$ and $L_{n, k-1}(p)$ used here is illustrated by Figure 21.

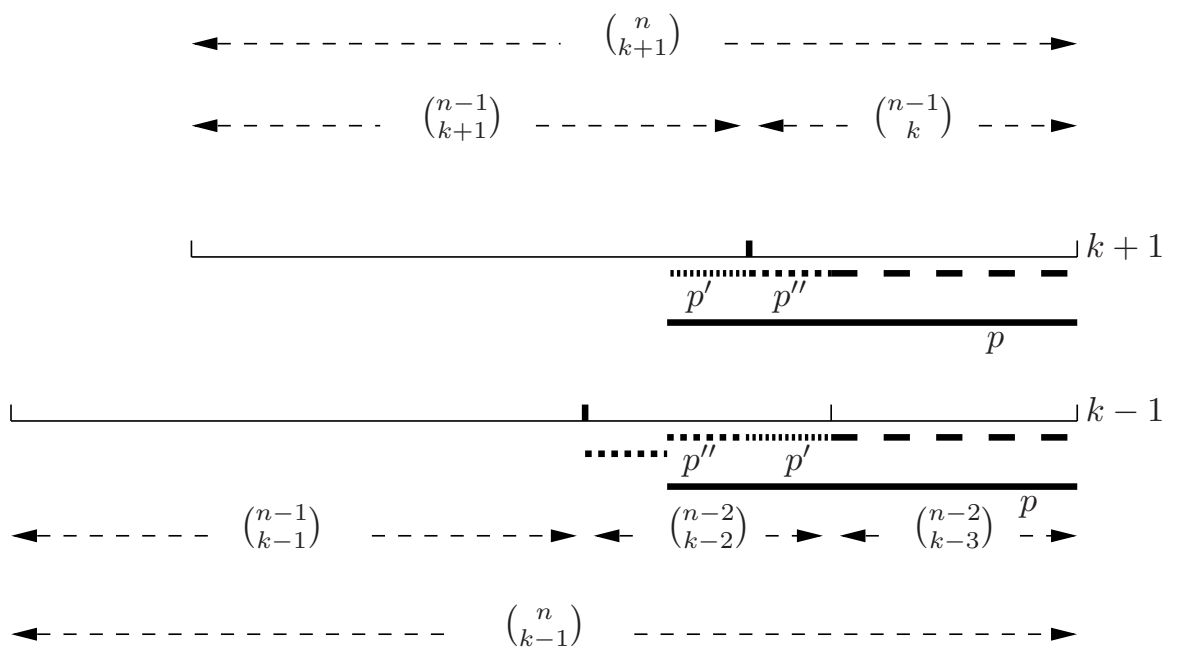

Figure 21: Partitioning of $L_{n, k+1}(p)$ and $L_{n, k-1}(p)$ in proving Proposition 5.3

Let $p=p^{\prime}+p^{\prime \prime}+\left(\begin{array}{l}n-2 \\ k-3\end{array}\right)$ be such that $\left(\begin{array}{c}n-1 \\ k\end{array}\right)=p^{\prime \prime}+\left(\begin{array}{l}n-2 \\ k-3\end{array}\right)$. Then $0<p^{\prime}+p^{\prime \prime} \leq\left(\begin{array}{l}n-2 \\ k-2\end{array}\right)$. It follows that $\left|\triangle_{N} L_{n, k+1}(p)\right|=\left|\triangle_{N} L_{n-1, k}\left(\left(\begin{array}{l}n-2 \\ k-3\end{array}\right)\right)\right|+\left|\triangle_{N} F_{n-1, k}\left(p^{\prime \prime}\right)\right|+\left|\triangle_{N} L_{n-1, k+1}\left(p^{\prime}\right)\right|$ 
and that

$$
\begin{aligned}
& \left|\nabla_{N} L_{n, k-1}(p)\right| \\
& \geq\left|\nabla_{N} L_{n-2, k-3}\left(\left(\begin{array}{l}
n-2 \\
k-3
\end{array}\right)\right)\right|+\left|\nabla_{N} L_{n-2, k-2}\left(p^{\prime}\right)\right|+\left|\nabla_{N} F_{n-2, k-2}\left(p^{\prime \prime}\right)\right| \\
& =\left|\nabla_{N} L_{n-1, k-2}\left(\left(\begin{array}{l}
n-2 \\
k-3
\end{array}\right)\right)\right|+\left|\nabla_{N} L_{n-1, k-1}\left(p^{\prime}\right)\right|+\left|\nabla_{N} F_{n-1, k-2}\left(p^{\prime \prime}\right)\right| .
\end{aligned}
$$

Proposition 5.3 then follows from IH 3.3.

Proposition 5.4. Let $n>32$ and $k>\frac{n}{2}$ with $\left(\begin{array}{c}n-2 \\ k-3\end{array}\right) \leq\left(\begin{array}{c}n-1 \\ k\end{array}\right)$. Then Theorem 1.5 holds for $\left(\begin{array}{l}n-1 \\ k-2\end{array}\right)<p \leq\left(\begin{array}{c}n \\ k+1\end{array}\right)$.

Proof. See Figure 22 for an illustration.

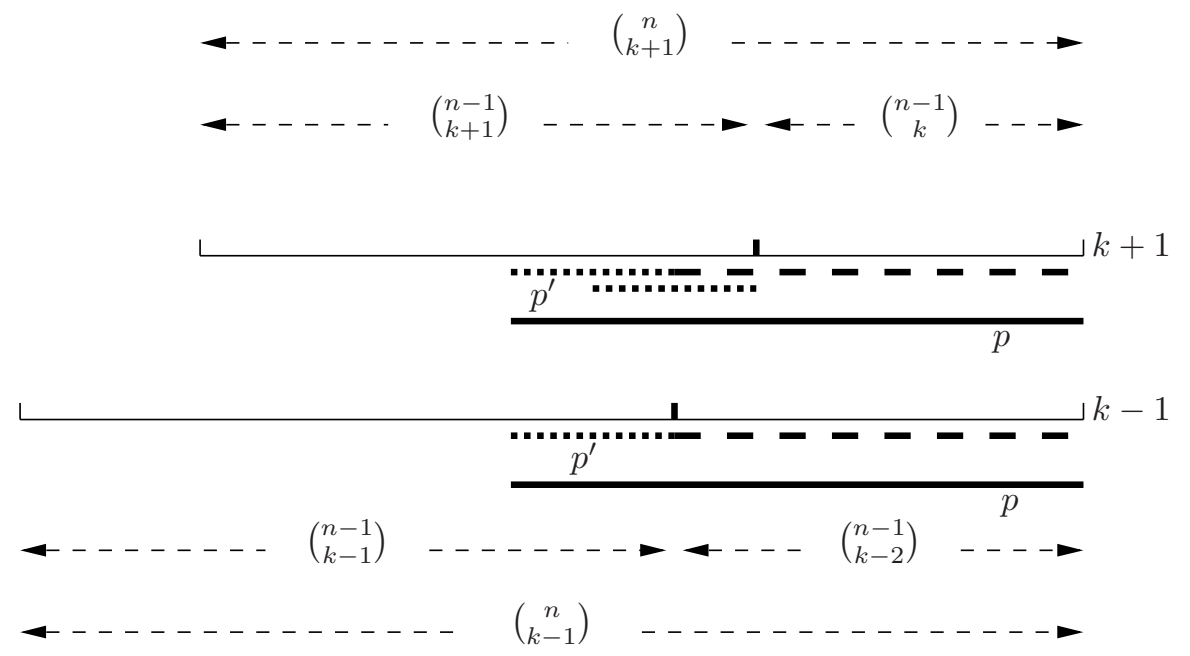

Figure 22: Partitioning of $L_{n, k+1}(p)$ and $L_{n, k-1}(p)$ in proving Proposition 5.4

Let $p=p^{\prime}+\left(\begin{array}{l}n-1 \\ k-2\end{array}\right)$, so $p^{\prime}>0$. Since $k>\frac{n}{2},\left(\begin{array}{c}n-1 \\ k-2\end{array}\right) \geq\left(\begin{array}{c}n-1 \\ k\end{array}\right)$. Thus, $\left|\triangle_{N} L_{n, k+1}(p)\right| \geq$ $\left|\triangle_{N} L_{n, k+1}\left(\left(\begin{array}{l}n-1 \\ k-2\end{array}\right)\right)\right|+\left|\triangle_{N} L_{n-1, k+1}\left(p^{\prime}\right)\right|$, and $\left|\nabla_{N} L_{n, k-1}(p)\right|=\left|\nabla_{N} L_{n, k-1}\left(\left(\begin{array}{l}n-1 \\ k-2\end{array}\right)\right)\right|+$ $\left|\nabla_{N} L_{n-1, k-1}\left(p^{\prime}\right)\right|$. Proposition 5.4 follows by IH 3.3 and Proposition 5.3 applied to $\left(\begin{array}{l}n-1 \\ k-2\end{array}\right)$.

This concludes the proof of Theorem 1.5. 


\section{Possible Alternative Proofs of Theorem 1.5}

The proof of Theorem 1.5 has been long but to date no other proof is known. The values $\left|\triangle F_{n, k+1}(p)\right|$ and $\left|\nabla_{N} F_{n, k-1}(p)\right|$ can be directly computed from the $(k+1)$-binomial and the $(k-1)$-binomial representation of $p$ respectively (see $[1,11])$. These two binomial representations of $p$ are independent of $n$. This suggests that to prove Theorem 1.5 an induction on $p$ would be appropriate, although this seems to be difficult.

Another approach is to consider the real binomial representation of $p$ (see [12, pp. 81 \& 459]). This representation may be more convenient to use in investigating sizes of newshadows and new-shades. While computing $\left|\triangle F_{n, k+1}(p)\right|$ can be simplified by using this approach, a suitable expression for $\left|\nabla_{N} F_{n, k-1}(p)\right|$ seems hard to find.

The output (not given in this paper) of the algorithm used to prove that Theorem 1.5 holds for values of $n$ less than 33 shows that for $n$ fixed, the function $\left(\left|\nabla_{N} L_{n, k-1}(p)\right|+\left|\triangle_{N} L_{n, k+1}(p)\right|\right) / p$ seems to attain its minimum over all $k$ and $p$ when $k=\frac{n}{2}-1, n$ even, and when $k=\frac{n-1}{2}, n$ odd, and $n$ sufficiently large. A strategy to prove Theorem 1.5 could then be to prove that this is indeed the case, and to prove that Theorem 1.5 holds for $k=\frac{n}{2}-1, n$ even, and for $k=\frac{n-1}{2}, n$ odd, and $n$ sufficiently large. The latter seems reasonably easy to show, however the former appears difficult to prove.

\section{References}

[1] I. Anderson. Combinatorics of Finite Sets. Oxford Science Publications, 1987.

[2] B. Bollobás. Combinatorics. Cambridge University Press, 1986.

[3] G.F. Clements. A Minimization Problem Concerning Subsets of a Finite Set. Discrete Mathematics, 4:123-128, 1973.

[4] G.F. Clements. The Kruskal-Katona Method Made Explicit. Journal of Combinatorial Theory Series A, 21:245-249, 1976.

[5] G.F. Clements. More on the Generalised Macauley Theorem - II. Discrete Mathematics, 18:253-264, 1977.

[6] D.E. Daykin, J. Godfrey, and A.J.W. Hilton. Existence Theorems for Sperner Families. Journal of Combinatorial Theory, Series A, 17:245-251, 1974.

[7] G. Katona. A Theorem on Finite Sets. In Theory of Graphs, Proc. Colloq. Tihany, pages 187-207, New York, 1966. Akademiai Kiado, Academic Press.

[8] Á. Kisvölcsey. Flattening Antichains. Combinatorica, to appear. 
[9] J.B. Kruskal. The Number of Simplices in a Complex. In R. Bellman, editor, Mathematical Optimization Techniques, pages 251-78. University of California Press, Berkeley, 1963.

[10] P. Lieby. The Separation Problem. Honours thesis, Northern Territory University, Casuarina NT 0909, Australia, 1994.

[11] P. Lieby. Extremal Problems in Finite Sets. PhD thesis, Faculty of Science, Northern Territory University, Casuarina NT 0909, Australia, February 1999.

[12] L. Lovász. Combinatorial Problems and Exercises. North-Holland, 1979.

[13] F. Maire. On the Flat Antichain Conjecture. Australasian Journal of Combinatorics, 15:241-245, 1997.

[14] I.T. Roberts. Extremal Problems in Finite Sets. PhD thesis, Faculty of Science, Northern Territory University, Casuarina NT 0909 Australia, February 1999.

[15] E. Sperner. Ein Satz über Untermengen einer endligen Menge. Math. Z., 27:544-8, 1928. 